\title{
The radio luminosity function of radio-loud quasars from the 7C Redshift Survey
}

\author{
Chris J. Willott, Steve Rawlings, Katherine M. Blundell and Mark Lacy \\ Astrophysics, Department of Physics, Keble Road, Oxford, OX1 3RH, U.K.
}

13 August 2018

\begin{abstract}
We present a complete sample of 24 radio-loud quasars (RLQs) from the new 7C Redshift Survey. Every quasar with a low-frequency (151 MHz) radio flux-density $S_{151}>0.5 \mathrm{Jy}$ in two regions of the sky covering $0.013 \mathrm{sr}$ is included; 23 of these have sufficient extended flux to meet the selection criteria, 18 of these have steep radio spectra (hereafter denoted as SSQs). The key advantage of this sample over most samples of RLQs is the lack of an optical magnitude limit. By combining the 7C and $3 \mathrm{CRR}$ samples, we have investigated the properties of RLQs as a function of redshift $z$ and radio luminosity $L_{151}$.

We derive the radio luminosity function (RLF) of RLQs and find that the data are well fitted by a single power-law with slope $\alpha_{1}=1.9 \pm 0.1$ (for $H_{\circ}=50 \mathrm{~km} \mathrm{~s}^{-1} \mathrm{Mpc}^{-1}$, $\left.\Omega_{\mathrm{M}}=1, \Omega_{\Lambda}=0\right)$. We find that there must be a break in the RLQ RLF at $\log _{10}\left(L_{151} /\right.$ $\left.\mathrm{W} \mathrm{Hz}{ }^{-1} \mathrm{sr}^{-1}\right) \lesssim 27$, in order for the models to be consistent with the $7 \mathrm{C}$ and $6 \mathrm{C}$ source counts. The $z$-dependence of the RLF follows a one-tailed gaussian which peaks at $z=1.7 \pm 0.2$. We find no evidence for a decline in the co-moving space density of RLQs at higher redshifts.

A positive correlation between the radio and optical luminosities of SSQs is observed, confirming a result of Serjeant et al. (1998). We are able to rule out this correlation being due to selection effects or biases in our combined sample. The radiooptical correlation and best-fit model RLF enable us to estimate the distribution of optical magnitudes of quasars in samples selected at low radio frequencies. We conclude that for samples with $S_{151} \lesssim 1$ Jy one must use optical data significantly deeper than the POSS-I limit $(R \approx 20)$, in order to avoid severe incompleteness.
\end{abstract}

Key words: Galaxies: active - quasars: general - quasars: emission lines

\section{INTRODUCTION}

The variation of the space densities and properties of quasars with redshift contains information about structure formation and evolution at different epochs. Radio galaxies are important for similar reasons and their relationship with radio-loud quasars (RLQs) is now under scrutiny. According to orientation-based unified schemes, RLQs and powerful radio galaxies are the same type of objects, their apparently different properties being due to a systematic difference in the angle between our line-of-sight and the radio jet axis (e.g. Scheuer 1987, Antonucci 1993). In orientation-based unification, the broad line region and quasar nucleus are hidden in radio galaxies behind an optically-thick torus. If there is not a sharp cut-off in the optical depth of the torus, then objects close to the transition angle between radio galaxies and quasars may appear to be faint reddened quasars (Baker 1997).

One of the major problems in trying to understand the collective properties of a sample of objects is ensuring that the sample provides reliable information on the parent population. Particularly in astrophysics, care must be taken to minimise and understand selection effects. For example, because of the steepness of the radio luminosity function, there is a strong correlation between radio luminosity and redshift in the 3CRR sample (Laing, Riley \& Longair 1983, hereafter LRL). Hence, it is impossible to disentangle correlations with radio luminosity from those with evolution with a single flux-limited sample. Radio quasar samples selected at high-frequency contain mostly flat-spectrum quasars (FSQs, $\alpha_{\text {rad }} \leq 0.5$ 风, where $\alpha_{\text {rad }}$ is evaluated at $1 \mathrm{GHz}$ in the source rest-frame. The radio emission from these objects is often dominated by optically-thick radio cores, which are en-

(C) 0000 RAS 
hanced by Doppler boosting because their jet axes lie close to the line-of-sight. Therefore their total radio flux-densities (and hence probability of being above a given flux-limit) are orientation-dependent and often strongly time variable (e.g. Seielstad, Pearson \& Readhead 1983). In contrast, the radio emission from steep-spectrum quasars (SSQs, $\alpha_{\text {rad }}>0.5$ ) is dominated by optically-thin extended emission and hence low-frequency selected samples suffer far less greatly from these orientation-dependent or variability biases. Some radio sources, particularly compact-steep spectrum (CSS) sources (e.g. Fanti et al. 1995) and Giga-Hertz Peaked Spectrum (GPS) sources (e.g. O'Dea, Baum \& Stanghellini 1991), suffer synchrotron self-absorption at low-frequencies and hence have radio spectra which flatten at low-frequency, eg. 151 $\mathrm{MHz}$. By evaluating the spectral indices at rest-frame $1 \mathrm{GHz}$ we avoid rejecting these source from our sample, although the sensitivity of a survey to such sources is clearly a function of both survey frequency and source redshift (see Blundell, Rawlings \& Willott 1998a (Paper IV) for a fuller discussion).

The luminosity function describes the co-moving density of a class of objects as a function of redshift and luminosity. For quasars, one can define the luminosity function in terms of the optical luminosity function (OLF), X-ray luminosity function (XLF), or the radio luminosity function (RLF). Most large quasar surveys have been selected using optical, and more recently X-ray, criteria so the quasar OLF and XLF are better constrained than the RLF. Boyle, Shanks \& Peterson (1988) showed that the OLF can be fitted with a broken power-law with the break luminosity evolving with redshift - an effect normally termed pure luminosity evolution (PLE). However, Hewett, Foltz \& Chaffee (1993) and Miller et al. (1993) showed that PLE was an inadequate model to explain the evolution of luminous quasars. Hawkins \& Véron (1995) explained the break in the power-law of Boyle et al. as due to incompleteness in their data. Hewett et al. proposed that the power-law index of the OLF steepens as the redshift increases. This is supported by the recent analysis of the quasar OLF by Goldschmidt \& Miller (1998). The space density of the most luminous quasars shows little or no evolution (Miller et al. 1993), whilst the optically fainter quasars show significant evolution (a factor of $\sim 50$ increase between $z \sim 0.5$ and $z \sim 2$ ). The XLF also appears to have a broken power-law form (Maccacaro et al. 1991; Della Ceca et al. 1992). Boyle et al. (1994) find the bestfitting model for the XLF is pure luminosity evolution for $z<1.6$ and a constant co-moving density at higher redshift. However, it must be remembered that these quasar samples selected in the optical and X-ray wavebands may be prone to selection effects. If, for example, many quasars are highly reddened (Webster et al. 1995), then this would greatly alter the shape and evolution of the OLF, and because of absorption by neutral hydrogen, the XLF.

One of the difficulties of determining the quasar RLF is that radio surveys typically contain mostly radio galaxies, so all sources must be reliably identified as quasar or radio galaxy. Dunlop \& Peacock (1990) derived the RLF separately for flat- and steep-spectrum radio sources. However, the high selection frequency $(2.7 \mathrm{GHz})$ of the samples used

\footnotetext{
$\star$ The convention for spectral index, $\alpha_{\mathrm{rad}}$, is that $S_{\nu} \propto \nu^{-\alpha_{\mathrm{rad}}}$, where $S_{\nu}$ is the flux density at frequency $\nu$.
}

in this work meant a large fraction of flat-spectrum sources in their study and hence the steep-spectrum RLF was more poorly constrained. The steep-spectrum RLF fit of Dunlop $\&$ Peacock includes both radio galaxies and steep-spectrum quasars. No attempt was made to separate these different types of object. Hence the steep-spectrum quasar RLF has not previously been well-constrained. Radio selected quasar samples usually have additional optical selection criteria, which can be a cause of incompleteness and selection biases. Since the $7 \mathrm{C}$ quasar sample has no optical selection criteria it is complete, so in conjunction with the 3CRR sample of LRL it can be used to derive the RLF for the low-frequency selected population which is dominated by SSQs.

There have been several suggestions of correlations between the radio and optical properties of AGN. The close relationship between narrow-line luminosity and lowfrequency radio luminosity (e.g. Baum \& Heckman 1989; Rawlings \& Saunders 1991; Willott et al. 1998b (Paper III)) suggests a physical link between these two properties. Correlations between the radio and optical luminosities of RLQs have been found (e.g. Browne \& Murphy 1987) but incompleteness worries mean that there are unknown selection effects which could affect the results. Correlations between the radio and optical may be due to orientation-dependent emission (e.g. Baker 1997). This is a problem for flat-spectrum RLQ samples because often the radio luminosities are dominated by a Doppler-boosted core and the optical luminosities too may therefore be orientation-dependent (e.g. Wills \& Lynds, 1978; Browne \& Wright 1985). Serjeant et al. (1998) presented evidence for a radio-optical correlation for SSQ samples. They used the 3CRR sample and a sample based on cross-matching the low-frequency (408 MHz) MRC radio catalogue with APM data - the MAQS sample. This sample has an optical magnitude limit as well as a radio flux limit. They also calculated $B$-band absolute magnitudes using observed $B$ magnitudes and $K$-corrections. For high-redshift quasars, this radiation was emitted in the UV and so will be greatly diminished if there is significant reddening of the quasar spectrum by dust.

Excepting 3CRR, all other SSQ samples also have optical magnitude limits, usually in the blue part of the spectrum (e.g. Vigotti et al. 1997; Riley et al. 1998). Hence faint (and/or reddened) quasars can be missed in these samples. If a substantial fraction of quasars are reddened then quasar samples with bright magnitude limits will miss them and an unrepresentative sample of the quasar population (i.e. only the 'naked' optically-bright ones) is seen. The only way to be sure of avoiding these selection effects is to identify all the radio sources in a flux-limited sample. However, this can be very time consuming because most radio samples are dominated by radio galaxies, not quasars.

Here we present a complete sample of 24 quasars from the new 7C Redshift Survey. In this survey we have obtained optical spectra of every radio source in two patches of sky (whose total area covers $0.013 \mathrm{sr}$ ), with a $151 \mathrm{MHz}$ flux density $S_{151}>0.5 \mathrm{Jy}$. We made high-resolution VLA maps of these sources to ensure reliable optical identifications. All 77 radio sources have optical / near-infrared identifications and only 6 lack secure spectroscopic redshifts. All 6 of these are galaxies with estimated redshifts from multi-colour photometry. Hence we can be confident that there is no significant incompleteness in our sample of radio-loud quasars. 
The layout of this paper (Paper II) is as follows. In Section 2 we present the data on the complete $7 \mathrm{C}$ quasar sample. In Section 3 we discuss our definition of a quasar in order to ensure that we are comparing similar types of objects from the $3 \mathrm{CRR}$ and $7 \mathrm{C}$ samples. This is necessary because the traditional definition of a quasar is no longer tenable in the light of unified schemes. The RLQ radio luminosity function is derived in Section 4 and compared with the optically-selected quasar OLF. In Section 5 we discuss the evidence for a correlation between the optical and radio luminosities of steep-spectrum quasars. In Section 6 we use our knowledge of the radio-optical correlation and the RLQ RLF to estimate the completeness of quasar samples selected with low-frequency radio and optical flux limits. The optical properties of the $7 \mathrm{C}$ quasar sample are to be discussed elsewhere (Willott et al. in prep., Paper VIII). Also in this forthcoming paper, we investigate the amount of reddening in the $7 \mathrm{C}$ quasar sample and its impact on the completeness of quasar surveys.

We assume throughout that $H_{\circ}=50 \mathrm{~km} \mathrm{~s}^{-1} \mathrm{Mpc}^{-1}$. All the calculations in this paper were done for three different cosmological models to account for the current uncertainty in the values of the matter density parameter $\left(\Omega_{\mathrm{M}}\right)$ and the cosmological constant parameter $\left(\Omega_{\Lambda}\right)$. The models considered are I: $\Omega_{\mathrm{M}}=1, \Omega_{\Lambda}=0$; II: $\Omega_{\mathrm{M}}=0, \Omega_{\Lambda}=0$; III: $\Omega_{\mathrm{M}}=0.1, \Omega_{\Lambda}=0.9$.

\section{THE 7C REDSHIFT SURVEY}

Only brief details of the 7C Redshift Survey are given here; full details will appear elsewhere (see also Rawlings et al. 1998; Willott et al. 1998a; Blundell et al. 1998b, 1998c (Paper I). The 7C survey was carried out with the Cambridge Low Frequency Synthesis Telescope (CLFST) at 151 $\mathrm{MHz}$ (McGilchrist et al. 1990) at a resolution of $70 \times 70$ $\operatorname{cosec}(\mathrm{Dec})$ arcsec. The $7 \mathrm{C}$ Redshift Survey was pursued in three separate patches of sky: $7 \mathrm{C}-\mathrm{I}, 7 \mathrm{C}-\mathrm{II}$ and $7 \mathrm{C}-\mathrm{III}$. In this paper we consider the $7 \mathrm{C}-\mathrm{I}$ and $7 \mathrm{C}-\mathrm{II}$ regions, which were chosen by Rossitter (1987) to overlap with the 5C6 and 5C7 fields of Pearson \& Kus (1978), respectively. The 7CIII region is centred on the North Ecliptic Pole (overlapping with the $38 \mathrm{MHz}$ selected sample of Lacy et al. 1993) and has similar spectroscopic completeness to $7 \mathrm{C}-\mathrm{I}$ and $7 \mathrm{C}-\mathrm{II}$. However the 7C-III objects lie above the declination limit of the UKIRT and we have been unable to obtain $K$-band images of most of these objects. For this reason the $7 \mathrm{C}-\mathrm{III}$ region will not be considered further in this paper. The $7 \mathrm{C}-$ I and 7C-II $151 \mathrm{MHz}$ data were subsequently re-analysed (and in the case of the 5C6 field re-observed) by Julia Riley. VLA data, where available, were retrieved from the archive (by kind permission of Barry Clark), or were recently obtained by us: full details of all these radio observations and the sample selection are described by in Paper I. The $7 \mathrm{C}-\mathrm{I}$ region covers an area of 0.0061 sr and contains 37 sources with $S_{151}>0.51 \mathrm{Jy}$. The $7 \mathrm{C}-\mathrm{II}$ region covers an area of 0.0069 sr and contains 40 sources with $S_{151}>0.48 \mathrm{Jy}$. All the sources in the final sample have now been mapped with the VLA to produce high-resolution radio maps. These are necessary to resolve the lobes and core and get an accurate position for the expected optical counterpart. The angular separations between the $7 \mathrm{C}$ radio source positions and the optical positions of the quasars are listed in Table 1 . Note that these separations are less than 10 arcsec in all cases (see Riley et al. 1998 for further discussion of this point).

Despite the low selection frequency of the $7 \mathrm{C}$ survey (151 MHz), it is possible that there could be Dopplerboosted quasars in the sample whose extended flux alone would not exceed the sample flux limit. By careful spectral fitting of the data, using integrated flux-densities at a number of frequencies from many radio surveys and our own VLA maps, we evaluated $1 \mathrm{GHz}$ rest-frame spectral indices (hereafter $\alpha_{\mathrm{rad}}$ ) for all the sources (as described in Blundell et al. 1998a). By consideration of these radio spectra and by examining our own VLA maps (Paper I), we were able to identify any $7 \mathrm{C}$ sources which exceeded the flux limit only because of the contribution of their Doppler boosted cores. Although six of the 24 quasars in the $7 \mathrm{C}$ sample have $\alpha_{\text {rad }} \leq 0.5$, we believe that just one $(5 \mathrm{C} 7.230)$ of these is promoted into the sample by such orientation-dependent radio emission. The other five quasars in the $7 \mathrm{C}$ sample with $\alpha_{\text {rad }} \leq 0.5$ we believe belong to a class of objects we term Core-JetS sources (CJSs); the details of their radio structure and spectra are discussed in Paper I, but a summary of their properties is as follows: these are quite compact sources, whose spectra may be showing evidence of synchrotron selfabsorption, which when resolved with MERLIN or VLBI reveal jet-type structures typically on both sides of the core.

We will use $237 \mathrm{C}$ RLQs in much of the subsequent analysis, comprising 18 which are SSQs (with $\alpha_{\text {rad }}>0.5$ ), and 5 which have extended flux which exceeds the flux-limit but which have $\alpha_{\text {rad }} \approx 0.4$.

A similar analysis of the 3CRR sample (see Appendix B) leads to a sample of RLQs consisting of 39 SSQs and one CJS (3C286). Possible reasons for the differing SSQ:CJS ratios in the two samples will be explored elsewhere. Two quasars from 3CRR (3C345 and 3C454.3) are excluded because they are promoted into the sample by Doppler boosted core emission.

\subsection{Source identification and $K$-band imaging}

All $7 \mathrm{C}-\mathrm{I}$ and $7 \mathrm{C}-\mathrm{II}$ objects have been imaged at $K$-band $(2.2 \mu \mathrm{m})$ for as long as required to obtain good signal-tonoise on the radio source identification (except the Dopplerboosted flat-spectrum quasar, 5C7.230). These images and their analysis will be published elsewhere (Willott et al, in prep), but a log of the observations is given in Table 1. Finding charts for the entire 7C Redshift Survey were generated from the APM catalogue at the RGO, Cambridge (Irwin, Maddox \& McMahon 1994). This catalogue utilises scanned plates from the Palomar Observatory Sky Survey (POSS). The astrometry of all images was fixed with the IRAF gasp package using these finding charts. $K$ magnitudes were measured with the IRAF apphot package using circular apertures of 5 arcsec diameter where possible. The infrared counterpart of the radio source was observed in every single image. Hence, we are confident that we have completely identified all members of the 7C Redshift Survey. The measured quasar $K$-magnitudes are listed in Table 3. 


\begin{tabular}{|c|c|c|c|c|c|c|}
\hline Name & Date & $\begin{array}{l}\text { Exposure } \\
\text { Time (s) }\end{array}$ & $\begin{array}{l}\text { Seeing } \\
(\operatorname{arcsec})\end{array}$ & \multicolumn{2}{|c|}{$\begin{array}{c}\text { Optical/NIR Position } \\
\text { (B1950.0) }\end{array}$} & $\begin{array}{c}\text { 7C-optical } \\
\text { separation }(\operatorname{arcsec})\end{array}$ \\
\hline $5 \mathrm{C} 6.5$ & 97Jan30 & 240 & 1.0 & 020414.40 & +332220.1 & 3.4 \\
\hline $5 \mathrm{C} 6.8$ & 97Jan30 & 240 & 1.0 & 020439.63 & +313751.7 & 1.6 \\
\hline $5 \mathrm{C} 6.33$ & 97Jan29 & 540 & 1.0 & 020836.32 & +333418.5 & 2.8 \\
\hline $5 \mathrm{C} 6.34$ & 96Sep04 & 540 & 1.0 & 020838.50 & +312130.2 & 3.6 \\
\hline $5 \mathrm{C} 6.39$ & 96Jan21 & 1080 & 1.1 & 020853.76 & +321446.1 & 2.5 \\
\hline $5 \mathrm{C} 6.95$ & 96Sep04 & 540 & 1.1 & $02 \quad 1149.24$ & +293640.0 & 8.3 \\
\hline $5 \mathrm{C} 6.160$ & 97Jan29 & 540 & 0.9 & 021444.80 & +332115.0 & 0.9 \\
\hline $5 \mathrm{C} 6.237$ & 97Jan29 & 540 & 1.1 & 021749.86 & +322723.3 & 2.0 \\
\hline $5 \mathrm{C} 6.251$ & 97Jan29 & 540 & 1.1 & 021850.44 & +340238.9 & 4.7 \\
\hline $5 \mathrm{C} 6.264$ & 97Jan29 & 540 & 0.9 & 021949.07 & +334341.3 & 2.2 \\
\hline $5 \mathrm{C} 6.282$ & 96Sep04 & 540 & 1.0 & 022140.78 & +340609.2 & 0.7 \\
\hline $5 \mathrm{C} 6.286$ & 97Jan30 & 360 & 1.1 & 022209.32 & +314549.2 & 4.4 \\
\hline $5 \mathrm{C} 6.287$ & 96Sep04 & 540 & 0.9 & $02 \quad 2219.95$ & +313900.6 & 4.5 \\
\hline $5 \mathrm{C} 6.288$ & 95Mar01 & 1080 & 1.1 & $02 \quad 2219.33$ & +310527.5 & 1.1 \\
\hline $5 \mathrm{C} 6.291$ & 97Jan29 & 1080 & 1.1 & 022309.74 & +340800.6 & 0.6 \\
\hline $7 \mathrm{C} 0808+2854$ & 97Jan30 & 540 & 1.2 & 080832.11 & +285401.8 & 5.7 \\
\hline $5 \mathrm{C} 7.17$ & $96 \mathrm{Mar} 10$ & 540 & 1.1 & 080956.36 & +270057.5 & 6.2 \\
\hline $5 \mathrm{C} 7.70$ & 97Jan30 & 540 & 1.3 & 081400.93 & +292730.9 & 1.3 \\
\hline $5 \mathrm{C} 7.85$ & $96 \mathrm{Mar} 10$ & 540 & 1.2 & 081445.58 & +272600.7 & 1.1 \\
\hline $5 \mathrm{C} 7.87$ & $96 \operatorname{Mar} 10$ & 540 & 1.2 & 081503.89 & +245827.3 & 6.0 \\
\hline $5 \mathrm{C} 7.95$ & 95Mar01 & 1080 & 1.3 & 081520.01 & +244505.4 & 6.8 \\
\hline $5 \mathrm{C} 7.118$ & 96Mar10 & 540 & 1.2 & $\begin{array}{lll}08 & 16 & 15.06\end{array}$ & +265129.3 & 1.7 \\
\hline $5 \mathrm{C} 7.194$ & $96 \mathrm{Mar} 10$ & 540 & 1.0 & 081914.39 & +254809.8 & 0.9 \\
\hline $5 \mathrm{C} 7.195$ & 95Mar01 & 2520 & 1.0 & 081920.11 & +251537.8 & 0.8 \\
\hline $5 \mathrm{C} 7.230$ & - & - & - & 082134.26 & +244828.5 & 3.0 \\
\hline $7 \mathrm{C} 0825+2930$ & 97Jan30 & 240 & 1.3 & 082505.45 & +293017.9 & 3.2 \\
\hline
\end{tabular}

Table 1. Log of $K$-band observations of the $7 \mathrm{C}$ quasars and broad-line radio galaxies using IRCAM3 on the UKIRT. The observations on 96Sep04 and 96Mar10 were part of the UKIRT service observing program. All other observations were made by us. The 7C-optical separation is the angular separation (in arcsec) between the optical position and the radio position given in the $7 \mathrm{C}$ catalogue. The position of $5 \mathrm{C} 7.230$ is from its identification in the APM catalogue, since no $K$-band imaging of this object has been obtained.

\subsection{Optical spectroscopy}

About half of the $7 \mathrm{C}-\mathrm{I} / 7 \mathrm{C}-\mathrm{II}$ quasars (13 out of 24 ) were identified on the POSS-I plates as the likely optical counterpart of the radio source, prior to spectroscopy. Some of the other quasars were identified from prior imaging as candidate identifications. Where the object was identified, an optical spectrum was taken at the position of this object. If an object was not identified before time with a spectrograph was available, then a spectrum was taken using blind offsetting to the radio position, aligning the slit with the radio axis (Rawlings, Eales \& Warren 1990). Most spectra were obtained with the ISIS long-slit spectrograph at the WHT. Table 2 lists the details of the quasar spectroscopy. The atmospheric conditions in January 1994 were not photometric since clouds affected the transparency.

The spectra were reduced in the IRAF package by subtracting the bias, flat-fielding with a combination of twilight sky and tungsten lamp spectra, wavelength calibration with comparison arc lamps, extinction correction, background subtraction and flux-calibration using spectrophotometric standards. One-dimensional spectra were extracted from the 2-D frames using a FWHM aperture for a good signal-to-noise ratio (snr) and a FWZI aperture for flux measurements. Atmospheric absorption was corrected for by scaling the absorption from a standard star spectrum by the airmass of the quasar observation. Distortions where the red and blue spectra meet (due to the dichroic) were also corrected using the flux-standard spectra. Final wavelength calibration was achieved by applying a shift to the zero-point from the $5577 \AA$ skyline. Cosmic rays were identified in the $2-\mathrm{D}$ spectra and edited out of the $1-\mathrm{D}$ spectra. Finally, the red and blue spectra were joined together, averaging over 50 $\AA$ where the snr of the spectra were approximately equal.

The final FWHM-aperture spectra are shown in Appendix A. A gaussian fit was made to the lines to determine the line centres and FWHMs. Where there was significant absorption present in the line profile, this was removed before the fit. The redshift of each quasar was determined from narrow line positions if possible, because these are more accurately measured and believed to be at the systemic redshift. Where narrow line centres could not be measured accurately, a weighted mean of broad line centres was used to determine the quasar redshift. For some objects blue-ward absorption of $\operatorname{Ly} \alpha$ occurred, which shifts the measured line centre to higher wavelengths. In these cases the redshift measured from Ly $\alpha$ was not used in the redshift estimation procedure. Line fluxes were measured with IRAF, by fitting a linear continuum and integrating the flux above this continuum. The observed equivalent widths were also measured in this way. The main source of error here is in accurately determining the underlying continuum. Appendix A contains the emission line data measured from the quasar spectra. 


\begin{tabular}{|c|c|c|c|c|c|c|c|}
\hline Name & $\begin{array}{c}\text { Telescope }+ \\
\text { Detector }\end{array}$ & Date & $\begin{array}{l}\text { Exposure } \\
\text { time }(\mathrm{s})\end{array}$ & $\begin{array}{l}\text { Slit width } \\
\text { (arcsec) }\end{array}$ & $\begin{array}{l}\text { Slit PA } \\
\left({ }^{\circ}\right)\end{array}$ & Notes & $\begin{array}{l}\text { Previous } \\
\text { reference }\end{array}$ \\
\hline $5 \mathrm{C} 6.5$ & WHT+ISIS & 94Jan09 & 600 & 2.0 & 75 & variable cloud cover & \\
\hline $5 \mathrm{C} 6.8$ & $"$ & 94Jan08 & 200 & 2.0 & 255 & variable cloud cover & \\
\hline $5 \mathrm{C} 6.33$ & $"$ & 95Jan30 & 1800 & 2.0 & 60 & & \\
\hline $5 \mathrm{C} 6.34$ & $"$ & 94Jan09 & 500 & 2.0 & 75 & variable cloud cover & \\
\hline $5 \mathrm{C} 6.39$ & $"$ & 97Jan09 & 1200 & 2.5 & 141 & & \\
\hline $5 \mathrm{C} 6.95$ & WHT+FOS-2 & 91Jan15 & 475 & 4.0 & 66 & & \\
\hline $5 \mathrm{C} 6.160$ & WHT+ISIS & 94Jan09 & 600 & 2.0 & 30 & variable cloud cover & \\
\hline $5 \mathrm{C} 6.237$ & $"$ & 94Jan08 & 300 & 2.0 & 255 & variable cloud cover & 1 \\
\hline $5 \mathrm{C} 6.251$ & $"$ & 94Jan08 & 300 & 3.0 & 255 & variable cloud cover & \\
\hline $5 \mathrm{C} 6.264$ & $"$ & 94Jan09 & 1200 & 2.0 & 30 & variable cloud cover & \\
\hline $5 \mathrm{C} 6.282$ & $"$ & 95Jan31 & 1300 & 2.0 & 81 & & \\
\hline $5 \mathrm{C} 6.286$ & $"$ & 94Jan09 & 600 & 2.0 & 30 & variable cloud cover & \\
\hline $5 \mathrm{C} 6.287$ & $"$ & 94Jan09 & 600 & 2.0 & 75 & variable cloud cover & \\
\hline $5 \mathrm{C} 6.288$ & $"$ & 95Jan31 & 1800 & 2.7 & 90 & & \\
\hline $5 \mathrm{C} 6.291$ & $"$ & 97Jan10 & 1800 & 1.0 & 24 & & \\
\hline $7 \mathrm{C} 0808+2854$ & $"$ & 97Apr07 & 600 & 2.5 & 72 & & 2 \\
\hline $5 \mathrm{C} 7.17$ & $"$ & 95Jan29 & 900 & 3.1 & 165 & & \\
\hline $5 \mathrm{C} 7.70$ & $"$ & 97Apr07 & 1200 & 1.5 & 120 & & \\
\hline $5 \mathrm{C} 7.85$ & $"$ & 94Jan10 & 730 & 2.0 & 280 & variable cloud cover & \\
\hline $5 \mathrm{C} 7.87$ & $"$ & 95Jan30 & 1700 & 3.1 & 60 & & \\
\hline $5 \mathrm{C} 7.95$ & $\mathrm{LICK}+\mathrm{KAST}$ & $960 \operatorname{Ct} 17$ & 600 & 2.0 & 143 & & \\
\hline $5 \mathrm{C} 7.118$ & WHT+ISIS & $97 \mathrm{Apr} 07$ & 300 & 2.5 & 137 & & \\
\hline $5 \mathrm{C} 7.194$ & $"$ & 97Apr07 & 300 & 2.5 & 172 & & \\
\hline $5 \mathrm{C} 7.195$ & $"$ & 95Feb01 & 1200 & 2.9 & 70 & & \\
\hline $5 \mathrm{C} 7.230$ & $"$ & 97Apr07 & 300 & 2.5 & 70 & & \\
\hline $7 \mathrm{C} 0825+2930$ & $"$ & 97Apr06 & 900 & 2.5 & 170 & & \\
\hline
\end{tabular}

Table 2. Log of spectroscopic observations of the quasars. The ISIS spectra cover the range from about $3000 \AA$ to $8500 \AA$ with resolution approximately $10 \AA$. The FOS-2 spectrograph at the WHT was used for 5 C6.95. The spectrum of $5 \mathrm{C} 7.95$ was kindly obtained for us with the Kast spectrograph on the 3-m Shane Telescope at Lick Observatory by Andrew Bunker and Isobel Hook. Redshifts for 5C6.237 and 7C0808+2854 had previously been determined in the literature . References: 1-Hook et al. (1996), 2-Barthel, Tytler \& Thomson (1990).

\subsection{Optical photometry}

$R$ and $b_{J}$ magnitudes have been measured for all the quasars and are listed in Table 3. If possible, these values were taken directly from the spectra. The FWZI aperture spectra were convolved with $R$ and $b_{J}$ filter transmission profiles. The flux within the filter bandwidth was then measured. A similar procedure was followed with the spectrophotometric standard, HD19445. This star has accurate photometry in the $b_{J}, R, I$ system (Gullixson et al. 1995). The measured quasar fluxes in each filter were then simply converted to a magnitude.

For the quasars observed in non-photometric conditions, this approach was not possible because a large fraction of the flux may be missing in the spectra. The variable cloud cover on these nights caused up to 2 magnitudes of extinction in the spectra. However, the majority of these quasars are detected on the APM $O$ and $E$ plates and have measured magnitudes. In one case, the quasar was only detected on the $O$ plate, so its $R$ magnitude was estimated from its optical spectral index and $b_{J}$ magnitude. Two of the faint quasars were imaged in $R$-band with the AUX port on the WHT, prior to spectroscopy. These were 5C6.33 and 5C6.39 which were observed on 1995 Jan 30 and 31, respectively. The exposure time for each image was $300 \mathrm{~s}$. A 300s $R$-band image of 5C6.264 was obtained at the $107^{\prime \prime}$ telescope at the Mc-
Donald Observatory, operated by the University of Texas at Austin, on 1995 Nov 20. These data were reduced using standard procedures, as described in Lacy \& Rawlings (1996).

\subsection{Galactic extinction corrections}

The $7 \mathrm{C}-\mathrm{I}$ and $7 \mathrm{C}-\mathrm{II}$ regions are at galactic latitudes, $b \approx$ $-30^{\circ}$ and $+30^{\circ}$, respectively. The amount of extinction and its variability across the sample was investigated. This is particularly important when one considers the intrinsic reddening of quasars. IRAS $100 \mu \mathrm{m}$ maps of the regions were obtained from Skyview (http://skyview.gsfc.nasa.gov/) and the positions of the $7 \mathrm{C}$ radio sources over-plotted. The $100 \mu \mathrm{m}$ surface brightness (corrected for zodiacal emission) in any direction is believed to be a very good indicator of the dust column density and therefore the visual extinction. The two variables are related by $A_{\mathrm{V}}=0.06 I_{100 \mu \mathrm{m}}$, where $I_{100 \mu \mathrm{m}}$ is in units of $\mathrm{MJy} \mathrm{sr}^{-1}$ (Rowan-Robinson et al. 1991).

The $100 \mu \mathrm{m}$ maps show significant structure across each region with several clumps of galactic cirrus with peak fluxes more than twice the mean value. The mean extinction in each region is $\left\langle A_{\mathrm{V}}\right\rangle=0.24(7 \mathrm{C}-\mathrm{I})$ and $\left\langle A_{\mathrm{V}}\right\rangle=0.19(7 \mathrm{C}-$ II). None of the quasars lie in the direction of the bright patches of cirrus. $5 \mathrm{C} 6.95$ is the quasar with the highest visual extinction value, $A_{\mathrm{V}}=0.32$. The difference between the $B$ - 


\begin{tabular}{|c|c|c|c|c|c|c|c|c|c|c|}
\hline Name & $\begin{array}{l}S_{151} \\
(\mathrm{Jy})\end{array}$ & $\begin{array}{c}\log _{10}\left(L_{151} /\right. \\
\left.\mathrm{WHz}^{-1} \mathrm{sr}^{-1}\right)\end{array}$ & $\alpha_{\mathrm{rad}}$ & $z$ & $\alpha_{\mathrm{opt}}$ & $K$ & $b_{J}$ & $R$ & $M_{B}$ & $\begin{array}{c}b_{J}, R \text { phot. } \\
\quad \text { source }\end{array}$ \\
\hline $5 \mathrm{C} 6.5$ & 0.767 & 26.432 & 0.96 & 1.038 & 0.1 & $16.26 \pm 0.05$ & $19.15 \pm 0.25$ & $18.28 \pm 0.25$ & -24.91 & 1,1 \\
\hline $5 \mathrm{C} 6.33$ & 0.570 & 26.585 & 0.96 & 1.496 & 0.7 & $18.23 \pm 0.19$ & $22.33 \pm 0.17$ & $21.08 \pm 0.05$ & -23.20 & 2,3 \\
\hline $5 \mathrm{C} 6.34$ & 0.544 & 27.064 & 0.77 & 2.118 & 0.5 & $15.94 \pm 0.04$ & $18.55 \pm 0.25$ & $17.94 \pm 0.25$ & -26.65 & 1,1 \\
\hline $5 \mathrm{C} 6.39$ & 0.532 & 26.629 & 0.80 & 1.437 & 0.5 & $17.87 \pm 0.08$ & $22.00 \pm 0.15$ & $21.35 \pm 0.07$ & -23.09 & 2,3 \\
\hline $5 \mathrm{C} 6.237^{\ddagger}$ & 1.665 & 27.106 & 0.36 & 1.620 & 0.8 & $15.76 \pm 0.03$ & $18.58 \pm 0.25$ & $17.24 \pm 0.25$ & -26.58 & 1,1 \\
\hline $5 \mathrm{C} 6.251$ & 0.561 & 26.776 & 0.94 & 1.665 & 0.1 & $17.66 \pm 0.11$ & $19.35 \pm 0.25$ & $18.93 \pm 0.25$ & -24.84 & 1,1 \\
\hline $5 \mathrm{C} 6.264$ & 0.948 & 26.373 & 0.81 & 0.832 & $1.3^{*}$ & $16.16 \pm 0.04$ & $19.99 \pm 0.30$ & $19.49 \pm 0.04$ & -23.50 & 5,4 \\
\hline $5 \mathrm{C} 6.282$ & 0.605 & 27.068 & 0.84 & 2.195 & 0.6 & $18.20 \pm 0.18$ & $22.17 \pm 0.15$ & $21.70 \pm 0.14$ & -23.91 & 2,2 \\
\hline $5 \mathrm{C} 6.286$ & 0.676 & 26.720 & 1.04 & 1.339 & 0.1 & $17.62 \pm 0.20$ & $19.83 \pm 0.35$ & $19.20 \pm 0.35$ & -24.34 & 1,1 \\
\hline $5 \mathrm{C} 6.287$ & 1.565 & 27.601 & 1.14 & 2.296 & 0.0 & $16.11 \pm 0.04$ & $18.69 \pm 0.25$ & $17.81 \pm 0.14$ & -26.74 & 1,1 \\
\hline $5 \mathrm{C} 7.85$ & 1.420 & 26.716 & 0.82 & 0.995 & 1.8 & $16.24 \pm 0.03$ & $20.68 \pm 0.23$ & $19.72 \pm 0.19$ & -23.74 & 2,2 \\
\hline $5 \mathrm{C} 7.87$ & 0.843 & 27.132 & 0.86 & 1.764 & 0.1 & $18.74 \pm 0.22$ & $21.47 \pm 0.16$ & $21.19 \pm 0.13$ & -23.28 & 2,2 \\
\hline $5 \mathrm{C} 7.95$ & 0.672 & 26.671 & 0.89 & 1.203 & 0.5 & $16.61 \pm 0.03$ & $20.07 \pm 0.25$ & $18.82 \pm 0.25$ & -24.73 & 1,1 \\
\hline $5 \mathrm{C} 7.118^{\dagger}$ & 1.417 & 26.174 & 0.69 & 0.527 & -0.1 & $15.75 \pm 0.03$ & $19.15 \pm 0.13$ & $19.06 \pm 0.11$ & -22.77 & 2,2 \\
\hline $5 \mathrm{C} 7.194$ & 1.701 & 27.301 & 0.75 & 1.738 & 0.2 & $15.91 \pm 0.03$ & $18.37 \pm 0.11$ & $18.10 \pm 0.10$ & -26.20 & 2,2 \\
\hline $5 \mathrm{C} 7.195$ & 0.889 & 27.174 & 0.90 & 2.034 & 0.6 & $17.71 \pm 0.05$ & $21.73 \pm 0.17$ & $21.27 \pm 0.15$ & -24.16 & 2,2 \\
\hline $5 \mathrm{C} 7.230^{\ddagger \ddagger}$ & 0.634 & 26.538 & 0.16 & 1.242 & 0.4 & - & $19.94 \pm 0.15$ & $19.35 \pm 0.13$ & -24.04 & 2,2 \\
\hline $7 \mathrm{C} 0825+2930^{\ddagger}$ & 0.719 & 26.989 & 0.45 & 2.315 & 1.1 & $17.55 \pm 0.15$ & $20.79 \pm 0.16$ & $20.63 \pm 0.14$ & -24.85 & 2,2 \\
\hline
\end{tabular}

Table 3. Basic data and photometry for the quasar sample. Radio luminosities and absolute magnitudes were calculated assuming $\Omega_{M}=1, \Omega_{\Lambda}=0 . K$ magnitudes are all measured using 5 arcsec diameter apertures except for 5C6.286, 5C6.288 \& 7C0825+2930 $(3$, 4 and 4 arcsec respectively, because of nearby objects). $b_{J}$ mags were calculated from $B$ mags using $B \approx b_{J}-0.14$ (e.g. Serjeant et al. 1998). $\alpha_{\mathrm{opt}}, b_{J}$ and $R$ values corrected for galactic reddening: $7 \mathrm{C}-\mathrm{I}, A_{B}=0.32, A_{R}=0.20 ; 7 \mathrm{C}-\mathrm{II}, A_{B}=0.25, A_{R}=0.16 .{ }^{*}$ The spectrum of $5 \mathrm{C} 6.264$ has a very poor snr because of several magnitudes of extinction by cloud and that of $5 \mathrm{C} 6.288$ only shows weak continuum because it is very faint. Therefore their optical spectral indices have been calculated assuming a power-law from their $R$ and $K$ magnitudes. ${ }^{\dagger} 5 \mathrm{C} 7.17 \& 5 \mathrm{C} 7.118$ are classified as broad-line radio galaxies (BLRGs), not quasars, because they have $M_{B}>-23$ (see Section 3). ${ }^{\ddagger}$ These 5 quasars are core-jets sources (CJS; see Section 2). ${ }^{\ddagger \ddagger} 5 \mathrm{C} 7.230$ is the only $7 \mathrm{C}$ quasar which does not have sufficient extended flux at $151 \mathrm{MHz}$ to be included as a member of the complete sample.

Key to $b_{J}, R$ photometry:

1 - APM catalogue

2 - measured from spectrum

3 - WHT AUX port R band image

4 - McDonald Observatory, University of Texas at Austin, 107" Telescope R band image

5 - used measured $R$ mag. and assume $b_{J}-R=0.5$ adopting $\alpha_{\mathrm{opt}}=0.0$

6 - not detected on the E plate, only on the O plate, assumed $b_{J}-R=0.5$ to calculate $R$ magnitude.

band extinction to this quasar and the mean in 5C6 is 0.1 mag. This is much less than typical magnitude errors (such as those from the APM), so we are justified in applying the same correction for objects in the same field.

The effect of galactic extinction on the spectra was also investigated. A flat spectrum $\left(\alpha_{\mathrm{opt}}=0\right)$ was created and then artificially reddened by various amounts (using the reddening curve of Savage \& Mathis 1979). It was found that there is a significant reddening of the initial flat spectrum. A power-law was fitted to the reddened spectra in the region 4000-6000 $\AA$. For the least reddened spectra (those in $7 \mathrm{C}-\mathrm{II})$, the reddened flat spectrum was fitted with a powerlaw with spectral index, $\alpha_{\mathrm{opt}}=0.22$. For $7 \mathrm{C}-\mathrm{I}, \alpha_{\mathrm{opt}}=0.27$ and the most heavily galactic reddened quasar in the sample, 5 C6.95, $\alpha_{\mathrm{opt}}=0.35$. Hence, the scatter in $\alpha_{\mathrm{opt}}$ due to variable galactic reddening is $< \pm 0.1$ and therefore not significant compared with the errors in the determination of $\alpha_{\text {opt }}$. The spectra shown in Figure A3 have not been dereddened. However, the values of $b_{J}, R$ and $\alpha_{\text {opt }}$ in Table 3 have been corrected for galactic extinction.

\section{OPTICAL CLASSIFICATION OF RADIO-LOUD AGN}

Historically, quasars were defined as luminous objects with unresolved 'stellar' IDs and strong, broad (FWHM > 2000 $\mathrm{km} \mathrm{s}^{-1}$ ) emission lines. In contrast, radio galaxies have a resolved optical appearance and narrow $(\mathrm{FWHM} \approx 1000$ $\mathrm{km} \mathrm{s}^{-1}$ ) or absent emission lines. However, it is now ap- 
parent that this simple classification scheme is inadequate. The increased sensitivity and resolution of optical and nearIR imaging reveals host galaxies around quasars, particularly at low $z$. Hence a weak quasar in a luminous galaxy will have a resolved optical counterpart. Weak, broad emission lines have also been seen in some radio galaxies. These objects are called broad-line radio galaxies (BLRGs) and have absolute magnitudes, $M_{B}>-23$. Spectropolarimetry of some radio galaxies has revealed broad lines scattered into our line-of-sight (e.g. 3C234: Antonucci 1984, Cygnus A: Ogle et al. 1997, 3C324: Cimatti et al. 1996), as expected in orientation-based unified schemes. It is also clear that some quasars have been reddened by dust so that the quasar is significantly dimmed in the UV (e.g. 3C22: Rawlings et al. 1995, 3C41: Simpson, Rawlings \& Lacy, in prep.). The identification of this type of reddened quasar suffers from a redshift bias, because at higher redshifts optical spectra sample shorter wavelengths where there is greater extinction. Near-infrared data is required to identify reddened quasars at high- $z$, e.g. the broad H- $\alpha$ line observed in the near-IR spectrum of 3C22 (Rawlings et al. 1995). Therefore the simple requirement that quasars have broad emission lines must be treated with caution, because even with light reddening this becomes dependent on redshift and whether NIR data is available.

Therefore we define quasars as objects with an unresolved nuclear source which itself has $M_{B}<-23\left(\Omega_{M}=1\right.$, $\Omega_{\Lambda}=0$ ) after eliminating light from host galaxies and other diffuse continuum (such as radio-aligned emission, e.g. McCarthy et al. 1987). This value is the same as the limiting absolute magnitude for quasars as defined in the quasar catalogue of Véron-Cetty \& Véron (1996). $M_{B}<-23$ was decided upon after plotting $M_{B}$ vs $L_{151}$ for all $3 \mathrm{CRR}$ and 7C Redshift Survey sources (see Section 5.1 for details of the $M_{B}$ calculation). There is a fairly clear separation between narrow-line only objects, which are less luminous than this, and objects with broad lines, which typically lie above $M_{B}=-23$. BLRGs fall below $M_{B}=-23$ and are consequently not classified as quasars. Further discussion of this classification scheme is deferred to Paper VIII.

Two members of the $7 \mathrm{C}$ Redshift Survey are in the BLRG category: 5C7.17 and 5C7.118. They will not be considered here as members of the complete quasar sample, although data is presented on them in Section 2. These objects are probably intrinsically weak AGN. They are not significantly dust-reddened, optically-luminous quasars because their optical-UV continua are blue (see Appendix A). The only remaining problem with this definition is that heavily reddened quasars $\left(A_{\mathrm{V}} \gg 1\right)$ will be excluded because their optical luminosities are diminished to below our limiting absolute magnitude. Such objects certainly exist (Simpson, Rawlings \& Lacy, in prep.) and the consequences of this will be discussed in detail in Paper VIII.

There are 2 sources in the $7 \mathrm{C}$ Redshift Survey which have optical luminosities $M_{B}<-23$ and which are spatially compact at about arcsec resolution, but for which we have no evidence of broad emission lines. We believe that both these objects are quasars and they are included as such in our sample. The spectrum of 5C6.264 was obtained in poor atmospheric conditions and we believe this is the reason that no broad lines are found in the spectrum. 5C6.288 is at high redshift $(z=2.982)$ and shows only narrow lines in its optical spectrum. These objects are briefly described in Appendix A and will be discussed in more detail in $\mathrm{Pa}$ per VIII. The optical spectrum of $5 \mathrm{C} 7.195$ also shows strong narrow lines with only a marginal broad component to the C III] emission line. This object is however known to be a lightly reddened $\left(A_{\mathrm{V}} \approx 1\right.$ ) quasar (Willott et al. 1998a and Paper VIII).

There are 8 objects traditionally classed as galaxies in the 3 CRR catalogue which have integrated absolute magnitudes, $M_{B}<-23$. All of these are in the range $-24<$ $M_{B}<-23$ and subtraction of the host galaxy luminosity and/or extended emission provides a limit on their unresolved optical component below our quasar definition value of $M_{B}$. The most luminous galaxy $3 \mathrm{C} 226$ has been shown by the HST imaging of Best, Longair \& Röttgering (1998) to contain a maximum point-source contribution of $21 \%$ (at the $90 \%$ confidence level). Therefore the maximum absolute magnitude of its nucleus is -22.0 , hence it is not by our definition a quasar.

As mentioned above, near-infrared observations of $3 \mathrm{C} 22$ and 3C41 (both listed by Spinrad et al. 1985 as radio galaxies) reveal reddened quasar nuclei. However, without de-reddening, these nuclei are fainter than our limit. Unified schemes predict that all powerful radio galaxies contain quasar nuclei which are heavily reddened. Therefore we must exclude all reddened quasars with observed $M_{B}>-23$ nuclei from our sample. 3C109 has often been quoted as a BLRG because at $z=0.3056$, its host galaxy is visible in optical images. However, its nucleus is brighter than our limit of $M_{B}=-23$, so we classify this source as a quasar. Goodrich \& Cohen (1992) estimate that there are $\simeq 3$ magnitudes of extinction towards the broad-line region of $3 \mathrm{C} 109$, so it is actually an intrinsically luminous quasar $\left(M_{B} \approx-26\right)$. It should also be noted here that some RLQs show significant time variability in their nuclear optical luminosities and this may lead to some uncertainty in object classification. The full list of 3CRR quasars and BLRGs is in Appendix B.

\section{RLQ RADIO LUMINOSITY FUNCTION}

We can use complete radio-selected quasar samples to derive the radio luminosity function (RLF) of radio-loud quasars. The dataset here is a combination of the 40 quasars in the $3 \mathrm{CRR}$ sample (Appendix B) and the $237 \mathrm{C}$ quasars with orientation-independent flux above the flux limit (as discussed in Section 2). The 3 flat-spectrum quasars (3C345, 3C454.3 \& 5C7.230) which are promoted into the samples by their prominent cores have not been included. Whilst this is only a small sample $(N=63)$, the simplicity of the selection criteria compensate. The $S_{178}>10.9 \mathrm{Jy}$ flux limit of the $3 \mathrm{CRR}$ sample was recalculated at the same selection frequency as the 7C survey (assuming low-frequency spectral index, $\left.\alpha_{151}=0.8\right)$, giving $S_{151}>12.43 \mathrm{Jy}$. The radio luminosities of the $3 \mathrm{CRR}$ and $7 \mathrm{C}$ sources were calculated at rest-frame $151 \mathrm{MHz}$ using the spectral fitting technique described in Blundell et al. (1998a). The radio luminosityredshift plane for both samples is shown in Figure 1. 


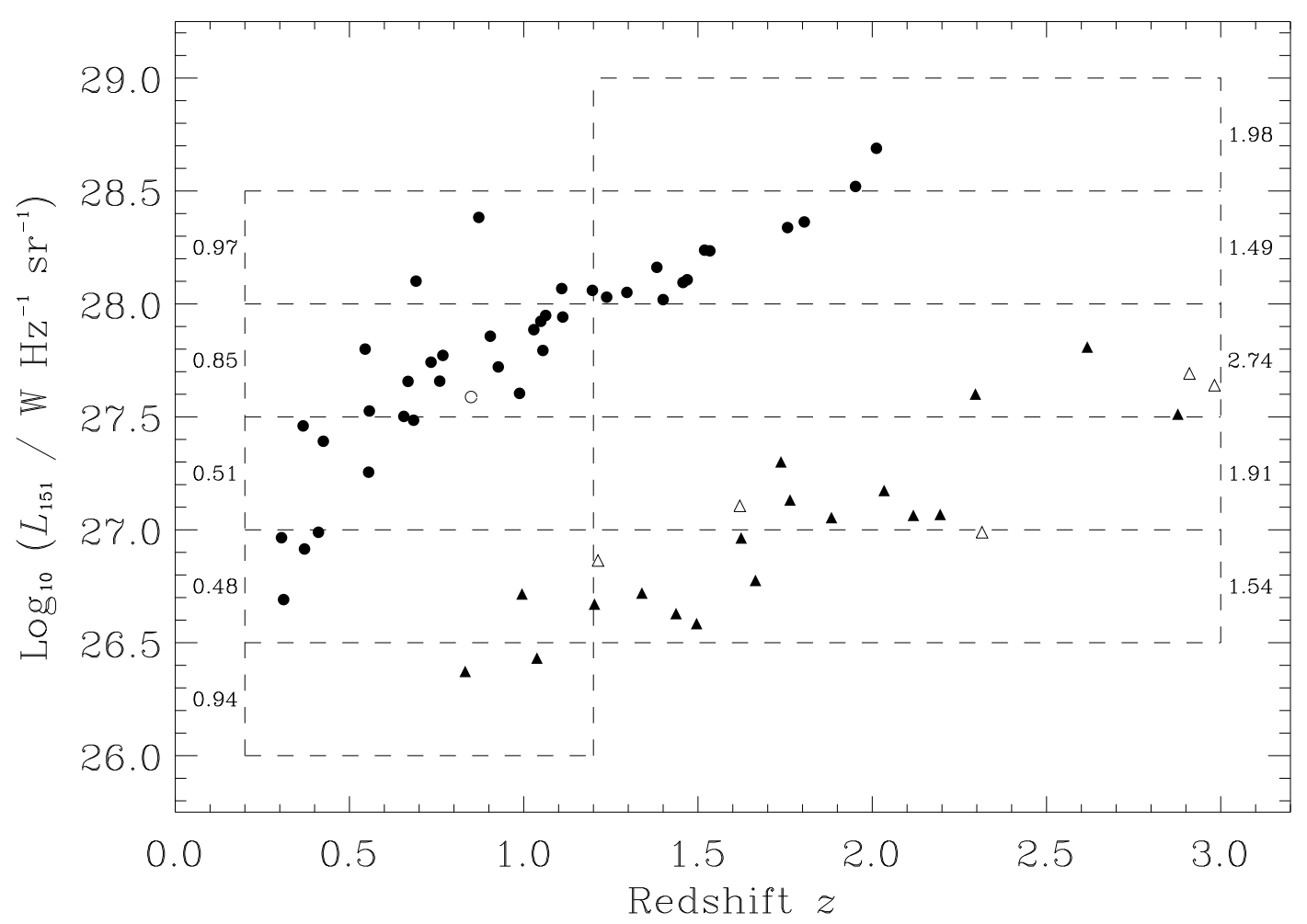

Figure 1. The radio luminosity-redshift plane for the 3CRR and $7 \mathrm{C}$ quasar samples. 3CRR quasars are plotted as circles; filled are SSQs, open is the CJS. The triangles are 7C quasars; filled are SSQs, open are CJSs. Note that the combination of 3CRR and 7C samples removes the strong luminosity-redshift correlation present in a single flux-limited sample. The bins in luminosity and redshift used in Section 4.1 are marked with dashed lines. Next to each bin is the mean redshift of the quasars in the bin. This plot is for cosmology I $\left(\Omega_{M}=1, \Omega_{\Lambda}=0\right)$.

\subsection{Binned $1 / V_{a}$ method}

The simplest way to estimate the luminosity function from a sample with known fluxes and redshifts is to use the binned $1 / V_{a}$ method as described in Boyle et al. (1987). $V_{a}^{j}$ is the co-moving volume available for a source $j$ in a bin with size $\Delta z$ (see Avni \& Bahcall, 1980, for more details). $\rho(L, z)$ is the number of sources per unit co-moving volume per unit $\log _{10} L_{151}$.

The RLF is given by

$\rho(L, z)=\sum_{j=1}^{N} \frac{1}{V_{a}^{j}}\left(\Delta \log _{10} L_{151}\right)^{-1}$.

From Marshall (1985), the error bars on each bin are given by

$\sigma=\left(\sum_{j=1}^{N}\left(V_{a}^{j}\right)^{-2}\right)^{0.5}\left(\Delta \log _{10} L_{151}\right)^{-1}$.

To reduce the effects of small number statistics, we chose to have two redshift bins, equal in $\Delta \log _{10}(1+z)$. The redshift bins are $0.2 \leq z \leq 1.2$ and $1.2<z \leq 3.0$. Each bin in luminosity is of size $\Delta \log _{10} L_{151}=0.5$. These bins are shown in Fig. 11.

The results of the binned analysis are shown in Fig. (for cosmology I). For each redshift bin, the RLF appears to be consistent with a single power-law. The slope of the luminosity function, $\alpha_{1}$, appears to steepen considerably from $\alpha_{1}=0.8$ in the low- $z$ bin to $\alpha_{1}=1.8$ in the high- $z$ bin. Marshall (1985) pointed out that with few redshift bins, there will be significant evolution across each bin. Since our coverage of the $L_{151}-z$ plane is non-uniform, this leads to the mean redshift of objects in high-luminosity bins usually being greater than that in the corresponding low-luminosity bins. Note there are exceptions to this, e.g. the two highest luminosity, high- $z$ bins contain only 3CRR quasars and consequently have lower mean redshifts than the lower luminosity bins containing only $7 \mathrm{C}$ quasars. The peak in mean redshift in the bin with the highest redshift $7 \mathrm{C}$ quasars is the cause of the apparent bump in the binned high- $z$ RLF. The mean redshifts of the sources in each bin are shown in Fig. 1. Due to the strong evolution from $z \approx 0$ to $z \approx 2$ of RLQs, the main effect of this is an artificial flattening of the power-law slopes in the binned method. Hence the slopes quoted above are probably lower limits to the real values.

For cosmology II, the binned luminosity function does not appear to be such a straight power-law as for cosmology I. The slope steepens slightly at high luminosities. This is most noticeable for the high- $z$ bins (but may be partly an artefact of the binning method as discussed above). For cos- 
mology III, the binned data has similar, straight slopes as for cosmology I.

\subsection{Parametric model fitting}

The binned analysis used in Section 4.1 is not good statistical use of complete sample data because information is lost when the objects are binned. This is especially important when the most information possible is to be extracted from a small dataset. An analytic model of the luminosity function, $\rho(L, z)$, is required. The model should have as few parameters as possible because of the small sample size being used to constrain it. The first simple model tested (model A) is a single power-law in luminosity with the redshift evolution specified by a gaussian, i.e.

$\rho(L, z)=\rho_{\circ}\left(\frac{L_{151}}{L_{\circ}}\right)^{-\alpha_{1}} \exp \left\{-\frac{\left(\frac{z-z_{\circ}}{z_{1}}\right)^{2}}{2}\right\}$,

where $\rho_{\circ}$ is a normalising term, $L_{\circ}=10^{27} \mathrm{~W} \mathrm{~Hz}^{-1} \mathrm{sr}^{-1}, \alpha_{1}$ is the power-law exponent, $z_{\circ}$ is the redshift of the gaussian peak and $z_{1}$ is the characteristic width of the gaussian. Thus there are only 4 free parameters in model A. The use of a gaussian redshift distribution is based on the observed redshift distribution of optically-selected quasars (Shaver et al. 1998) and the global star-formation rate (Madau et al. 1996).

To find the best-fit values of these parameters, the maximum likelihood method of Marshall et al. (1983) was used. $S$ is defined as $-2 \ln \mathcal{L}$, where $\mathcal{L}$ is the likelihood function and terms independent of the model parameters can be dropped. In this method, the aim is to minimise the value of $S$, which is given by:

$S=-2 \sum_{i=1}^{N} \ln \left[\rho\left(L_{i}, z_{i}\right)\right]+2 \iint \rho(L, z) \Omega(L, z) \frac{d V}{d z} d z d L$

where $(d V / d z) d z$ is the differential co-moving volume element, $\Omega(L, z)$ is the sky area available from the samples for these values of $L$ and $z$ and $\rho(L, z)$ is the model distribution being tested. In the first term of this equation, the sum is over all the $N$ sources in the combined sample. The second term is simply the integrand of the model being tested and should give $\approx 2 N$ for good fits. The integration range for the second term is $0<z<5,24<\log _{10} L_{151}<30$. Integrating the RLF up to $z=10$, instead of $z=5$, in the maximum likelihood fitting made no difference to any of the models, because the co-moving volume available becomes very small at high redshift. Initial starting values of the free parameters were estimated from the binned RLF. The parameters were optimised via the downhill simplex method (Press et al. 1992). Errors on the best-fit parameters were determined assuming a $\chi^{2}$ distribution of $\Delta S\left(\equiv S-S_{\min }\right)$ (Lampton, Margon \& Bowyer 1976). The error on each parameter was calculated by setting $\Delta S=1$ and allowing the other parameters to float. The errors quoted here are $68 \%$ confidence levels, as discussed in Boyle et al. (1988). Table 4 contains the best-fit parameters of all the models considered.

To assess the goodness-of-fit of a particular model the 2-dimensional Kolmogorov-Smirnov (KS) test was applied. This multi-dimensional version of the KS test was first proposed by Peacock (1983) and the version used is that of

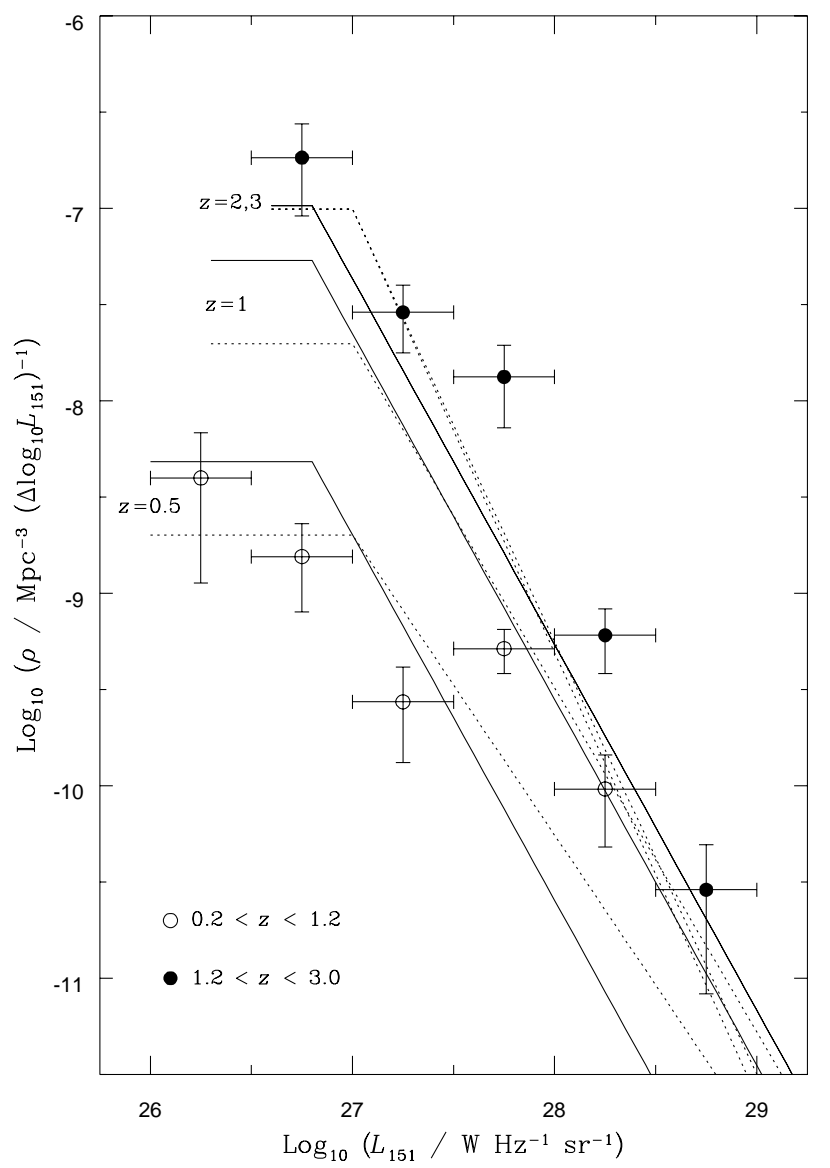

Figure 2. The radio luminosity function of RLQs derived here (for cosmology I). The points with $1 \sigma$ error bars are the binned RLF of Section 4.1. The solid lines are the maximum-likelihood best-fit model $\mathrm{C}$ and the dotted lines model $\mathrm{H}$, plotted for several values of $z$.

Press et al. (1992). It gives the probability, $P_{\mathrm{KS}}$, that the model distribution is a true representation of the dataset. Note that the KS test can only be used to reject models, not prove that they fit well. Indeed, for values of $P_{\mathrm{KS}} \gtrsim 0.2$ the probabilities may not be accurate and should not be used to distinguish between models. Therefore, we also evaluate the likelihood directly from the minimisation routine and use these to compare well-fitting models. For model A, $P_{\mathrm{KS}}=0.03$ for cosmology I and is even lower for cosmologies II \& III, which indicates a poor fit. Another way to check the goodness-of-fit of a RLF model is to use a Monte-Carlo simulation to create an artificial $L-z$ plane. These artificial datasets can then be compared to the actual $L-z$ plane for the 3CRR and 7C quasar samples. For model A, the simulated data contained too many high radio luminosity sources (i.e. in 3CRR) at both low $(z<0.3)$ and high $(2<z<3)$ redshift.

An additional constraint on our models down to $S_{151} \approx$ $0.1 \mathrm{Jy}$ comes from the $151 \mathrm{MHz}$ source counts from the $7 \mathrm{C}$ and 6C surveys (McGilchrist et al. 1990 and Hales, Baldwin \& Warner 1988, respectively). Since these counts include both quasars and radio galaxies, the source counts predicted from our quasar RLF models must be significantly lower. Al- 


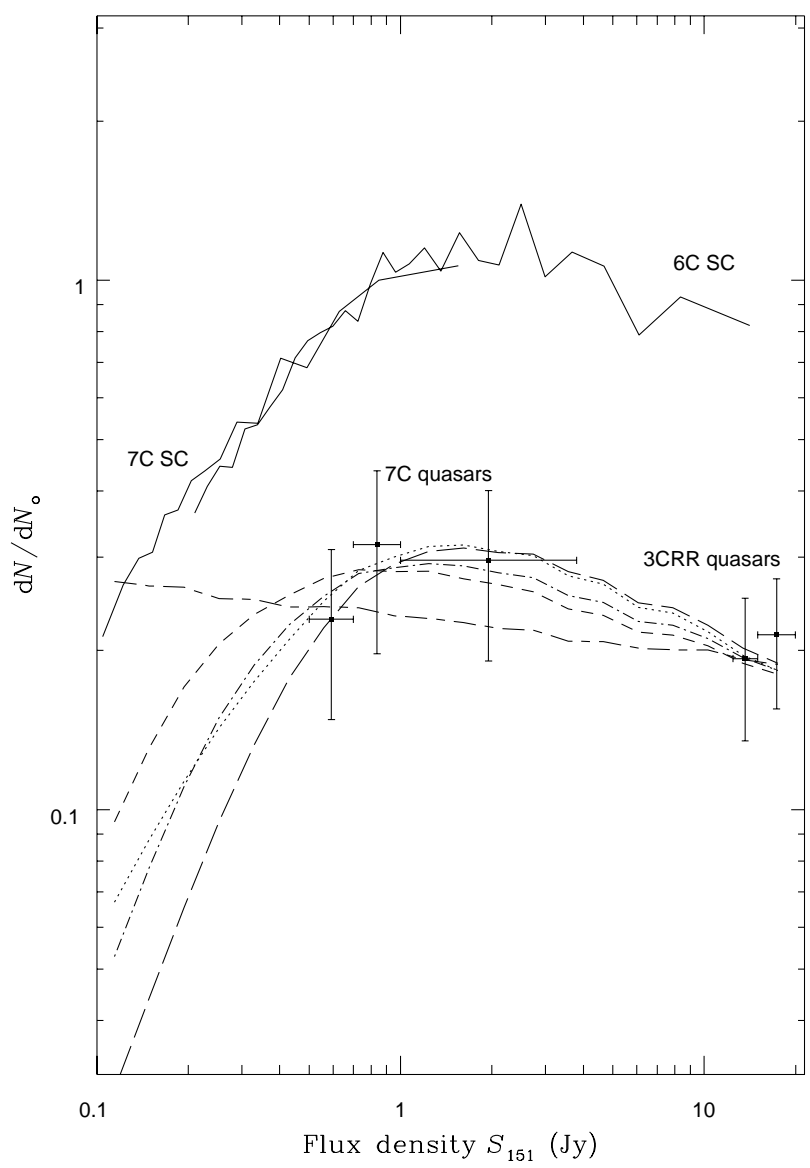

Figure 3. The normalised differential source counts from the $6 \mathrm{C}$ and $7 \mathrm{C}$ surveys are plotted here as solid lines and labelled 6C SC and $7 \mathrm{C} \mathrm{SC}$. Note that these counts include all radio sources, both quasars and radio galaxies. The solid circles with error bars show the binned quasar source counts from the $7 \mathrm{C}$ and 3CRR quasar samples described here. The source counts are normalised to the differential source counts for a uniform distribution in a Euclidean universe, such that $d N_{\circ}=2400\left(S_{\min }^{-1.5}-S_{\max }^{-1.5}\right)$, where $S_{\min }$ and $S_{\max }$ are the lower and upper flux limits of the bin. The other lines are quasar source count predictions from RLF models: A (short dash - long dash), B with $\log _{10}\left(L_{\text {break }}\right)=26.6$ (short dash), B with $\log _{10}\left(L_{\text {break }}\right)=26.8($ dot dash $)$, B with $\log _{10}\left(L_{\text {break }}\right)=$ 27.0 (long dash) and $\mathrm{C}$ with $\log _{10}\left(L_{\text {break }}\right)=26.8$ (dot).

though the fraction of quasars in complete samples at fluxes as low as $0.1 \mathrm{Jy}$ is unknown, one would not expect it to be vastly different from at $0.5 \mathrm{Jy}$. Models are considered consistent with the source counts if the percentage of quasars predicted at $0.1 \mathrm{Jy}$ is in the range $20 \%-40 \%$ (the quasar percentage in the $7 \mathrm{C}$ Redshift Survey $\left(S_{151} \geq 0.5 \mathrm{Jy}\right)$ is $\approx 30 \%$ ). The single power-law model (A) predicts that the RLQ source counts are greater than the total 7C source counts at $0.1 \mathrm{Jy}$. Figure 3 plots the $6 \mathrm{C}$ and $7 \mathrm{C}$ total source counts and the quasar source counts predicted by several RLF models. Taken together with the poor goodness-of-fit test from the $L-z$ data, the source counts constraint forces us to conclude that model A should be rejected.

The steep-spectrum RLF of Dunlop \& Peacock (1990) (which includes radio galaxies and quasars) could be fitted well by a broken power-law with the break at $\log _{10} L_{2.7} \approx$
25.5. This is equivalent to $\log _{10} L_{151} \approx 26.5$ (assuming $\left.\alpha_{\mathrm{rad}}=0.8\right)$. The over-prediction of the RLQ source counts by model A could be caused by the lack of a break in the luminosity function. This is because samples selected with $S_{151} \geq 0.1 \mathrm{Jy}$, would contain low luminosity sources $\left(\log _{10} L_{151} \approx 26\right)$ out to redshifts, $z \approx 1.5$. Therefore, the excess counts are due to the increased number density of low radio luminosity sources in models with an unbroken power-law.

Therefore, our next model (B) is a broken power-law in luminosity with a break at $L_{\text {break }}$. Unfortunately, we are unable to derive the value of $L_{\text {break }}$ or the power-law index below the break with our complete sample data, because we have so few quasars at these low radio luminosities. We assume that the RLF is flat $\left(\alpha_{1}=0\right)$ below the break. The value of $L_{\text {break }}$ can be estimated using the $6 \mathrm{C} / 7 \mathrm{C}$ source counts, because the counts at $0.1 \mathrm{Jy}$ are very sensitive to it. For cosmology I we can constrain $L_{\text {break }}$ for this model to $26.7<\log _{10}\left(L_{\text {break }}\right)<26.9$ (see Fig. 3). The quasar percentages for $\log _{10}\left(L_{\text {break }}\right)=26.6$ and $\log _{10}\left(L_{\text {break }}\right)=27.0$ are $45 \%$ and $15 \%$, respectively. $\log _{10}\left(L_{\text {break }}\right)=26.8$ was assumed since it gives a quasar fraction at $0.1 \mathrm{Jy}$ similar to that in the $7 \mathrm{C}$ sample considered here. Similarly $\log _{10}\left(L_{\text {break }}\right)=27.2$ and 27.3 for cosmologies II and III, respectively. Note that the value of $\log _{10}\left(L_{\text {break }}\right)$ is not a free parameter in our fits - it is constrained by the source counts, not the $L_{151}-z$ data. Maximum likelihood fits to the 4 free parameters of model B gave values of $P_{K S}$ in the range $0.4-0.7$ for all cosmologies which indicates that the model fits our data fairly well.

The functional form of the redshift evolution of the RLF in these models is symmetric in $z$ about $z_{0}$. This form was not based on theoretical predictions, but merely because it appears to be a good fit to the increase in density from $z=0$ to $z \approx 2$ (although clearly $\rho$ must decline at some high value of $z$ ). In the fit using model B above, $z_{\circ}=2.2$ (for cosmology I). There are only 6 quasars in the $3 \mathrm{CRR} / 7 \mathrm{C}$ sample with a redshift greater than this. Therefore, we cannot claim that there is a symmetric decline in the space density at $z>z_{0}$, just because we get a high probability from our goodnessof-fit test. To test whether a high redshift decline in $\rho$ is necessary, we repeated the model-fitting procedure using a one-tailed gaussian in $z$, such that $\rho(z)$ does not decline at all in the region $z_{\circ} \leq z \leq 5$ (and $\rho=0$ beyond $z=5$, as before). This new model (C) fits the data equally well with similar KS probabilities and a slightly greater likelihood. The peak redshift for $\mathrm{C}$ ranges from 1.7 for cosmology I to 2.0 for cosmology III. Hence we have no evidence for a decline in $\rho$ at $z \sim 2$ and we adopt model $\mathrm{C}$ as a slightly better fit to the data than $\mathrm{B}$.

Many derivations of the OLF and XLF have used the functional form $(1+z)^{k}$ to describe the redshift evolution of the quasar population (e.g. Boyle et al. 1994; Goldschmidt \& Miller 1998). We have replaced the gaussian redshift term from model A with this new term to make model D. Note that this reduces the number of free parameters in our fit from 4 to 3 . Model D provides a very poor fit. The KS probabilities are lower than $10^{-8}$ for all cosmologies. Monte-Carlo simulations of this model create many sources at low redshift and high luminosities, completely unlike the actual $L_{151}-z$ distribution. As before, we now include a low-luminosity break in the RLF (model E, analogous to model B). This 


\begin{tabular}{|c|c|c|c|c|c|c|c|c|c|c|c|c|}
\hline Model & Cos & $N_{\text {par }}$ & $\log _{10}\left(\rho_{\circ}\right)$ & $\alpha_{1}$ & $\alpha_{2}$ & $\log _{10}\left(L_{\text {break }}\right)$ & $z_{\circ}$ & $z_{1}$ & $k$ & $P_{\mathrm{KS}}$ & $\mathcal{L}$ & $\mathrm{Q} \%$ \\
\hline A & I & 4 & -7.199 & 1.585 & - & - & 2.01 & 0.505 & - & 0.03 & $10^{-7}$ & $>100$ \\
\hline $\mathrm{B}$ & I & 4 & -6.944 & 1.875 & - & 26.8 & 2.16 & 0.587 & - & 0.44 & 0.3 & 25 \\
\hline $\mathrm{C}$ & I & 4 & -7.150 & 1.900 & - & 26.8 & 1.66 & 0.436 & - & 0.49 & 1 & 35 \\
\hline $\mathrm{D}$ & I & 3 & -9.621 & 1.338 & - & - & - & - & 3.56 & $10^{-9}$ & $10^{-25}$ & 40 \\
\hline $\mathrm{E}$ & I & 3 & -9.276 & 2.105 & - & 27.2 & - & - & 3.97 & 0.006 & $10^{-10}$ & 40 \\
\hline $\mathrm{F}$ & I & 4 & -10.133 & 1.885 & - & 26.8 & 1.31 & - & 8.10 & 0.65 & 0.2 & 30 \\
\hline G & I & 5 & -6.629 & 1.334 & 0.416 & 27.0 & 2.49 & 0.647 & - & 0.69 & 6 & 25 \\
\hline $\mathrm{H}$ & I & 5 & -6.787 & 1.329 & 0.459 & 27.0 & 2.13 & 0.548 & - & 0.85 & 10 & 30 \\
\hline $\mathrm{A}$ & II & 4 & -7.225 & 1.576 & - & - & 2.02 & 0.511 & - & $10^{-4}$ & $10^{-8}$ & $>100$ \\
\hline $\mathrm{B}$ & II & 4 & -6.730 & 1.956 & - & 27.2 & 2.28 & 0.629 & - & 0.66 & 0.6 & 30 \\
\hline $\mathrm{C}$ & II & 4 & -6.897 & 1.976 & - & 27.2 & 1.84 & 0.495 & - & 0.58 & 1 & 35 \\
\hline $\mathrm{D}$ & II & 3 & -9.553 & 1.278 & - & - & - & - & 3.00 & $10^{-8}$ & $10^{-24}$ & 30 \\
\hline $\mathrm{E}$ & II & 3 & -8.416 & 2.308 & - & 27.6 & - & - & 3.96 & 0.03 & $10^{-7}$ & 70 \\
\hline $\mathrm{F}$ & II & 4 & -9.756 & 1.961 & - & 27.2 & 1.45 & - & 7.23 & 0.75 & 0.5 & 35 \\
\hline G & II & 5 & -6.302 & 1.657 & 0.261 & 27.4 & 2.69 & 0.699 & - & 0.44 & 0.8 & 30 \\
\hline $\mathrm{H}$ & II & 5 & -6.525 & 1.761 & 0.228 & 27.4 & 2.16 & 0.565 & - & 0.47 & 1.1 & 35 \\
\hline A & III & 4 & -7.334 & 1.558 & - & - & 2.06 & 0.508 & - & $10^{-5}$ & $10^{-8}$ & $>100$ \\
\hline B & III & 4 & -6.786 & 1.934 & - & 27.3 & 2.34 & 0.631 & - & 0.65 & 0.8 & 30 \\
\hline $\mathrm{C}$ & III & 4 & -6.936 & 1.946 & - & 27.3 & 1.99 & 0.531 & - & 0.84 & 1 & 35 \\
\hline $\mathrm{D}$ & III & 3 & -9.941 & 1.291 & - & - & - & - & 3.74 & $10^{-12}$ & $10^{-22}$ & 30 \\
\hline $\mathrm{E}$ & III & 3 & -8.704 & 2.212 & - & 27.7 & - & - & 4.31 & 0.05 & $10^{-6}$ & 60 \\
\hline $\mathrm{F}$ & III & 4 & -9.963 & 1.928 & - & 27.3 & 1.50 & - & 7.37 & 0.78 & 0.4 & 30 \\
\hline $\mathrm{G}$ & III & 5 & -6.262 & 1.590 & 0.285 & 27.5 & 2.83 & 0.703 & - & 0.51 & 1.1 & 30 \\
\hline $\mathrm{H}$ & III & 5 & -6.493 & 1.676 & 0.251 & 27.5 & 2.39 & 0.602 & - & 0.54 & 1.1 & 30 \\
\hline
\end{tabular}

Errors for model C (cosmology I): $\log _{10}\left(\rho_{\circ}\right) ;-0.12,+0.13, \alpha_{1} ;-0.09,+0.09, z_{\circ} ;-0.19,+0.22, \quad z_{1} ;-0.06,+0.07$.

Table 4. Best-fit parameters for model RLQ RLFs, as described in Section 4.2. Cos is the cosmological model assumed, $N_{\text {par }}$ is the number of free parameters in the fit, $\mathcal{L}$ is the likelihood from the fit, relative to model $\mathrm{C}$ for each cosmology and $\mathrm{Q} \%$ is the quasar percentage predicted at $0.1 \mathrm{Jy}$ from the source counts. A brief description of each model is given below (see Section 4.2 for more details). A: single power-law RLF, 2-tailed gaussian $z$ dependence.

B: single power-law RLF with low luminosity break, 2-tailed gaussian $z$ dependence.

C: single power-law RLF with low luminosity break, 1-tailed gaussian $z$ dependence.

D: single power-law RLF, $(1+z)^{k} z$-dependence.

E: single power-law RLF with low luminosity break, $(1+z)^{k} z$ dependence.

F: single power-law RLF with low luminosity break, $(1+z)^{k} z$ dependence with $z_{\max }=z_{o}$.

G: $z$ dependent power-law RLF with low luminosity break, 2-tailed gaussian $z$ dependence.

$\mathrm{H}: z$ dependent power-law RLF with low luminosity break, 1-tailed gaussian $z$ dependence.

model also fits poorly with $P_{\mathrm{KS}}<0.05$ for all cosmologies. The relative likelihoods between model $\mathrm{E}$ and model $\mathrm{C}$ are $<10^{-6}$. The low-luminosity break in model $\mathrm{E}$ is required to be 0.4 dex greater than models B \& $\mathrm{C}$ and even then the percentage of quasars in the source counts at $0.1 \mathrm{Jy}$ are $>50 \%$. Hence model E must be rejected. The reason for its poor fit is that the number density of quasars continues increasing up to high redshifts. This is illustrated by the Monte-Carlo simulations which show many sources at $z>3$, unlike the real data.

Boyle et al. (1994) find a good fit to the XLF with $(1+z)^{k}$ evolution if one allows a maximum redshift for this evolution, $z_{0}$, such that $\rho$ is constant at $z>z_{0}$. This should solve the problem of model $\mathrm{E}$ - too many high redshift quasars. Model F implements this form for the evolution and consequently there are now 4 free parameters in the fit. Model $\mathrm{F}$ is now extremely similar to model $\mathrm{C}$, in that in both $\rho$ increases up to $z_{\circ}$ and then remains constant. The fits for model $\mathrm{F}$ do indeed have similar parameter values to model $\mathrm{C}$. The $\mathrm{KS}$ probabilities are in the range 0.6 - 0.8. Model F agrees well with the source counts (with $\log _{10}\left(L_{\text {break }}\right)=26.8$, as for model C) and Monte-Carlo sim- ulations. The relative likelihoods of model $\mathrm{F}$ to model $\mathrm{C}$ are a factor of 2 to 5 lower, so we retain model $\mathrm{C}$ as the best-fitting model.

Our binned RLQ RLF has a power-law in $L_{151}$ which apparently steepens as the redshift increases. Therefore, the next logical step is to include this possibility into our RLF (model G). The power-law part of the model RLF is now

$\left(\frac{L_{151}}{L_{\circ}}\right)^{-\left(\alpha_{1}+\alpha_{2} z\right)}$

for $L_{151} \geq L_{\text {break }}$ and a flat power-law $\left(\alpha_{1}=0\right)$ for $L_{151}<L_{\text {break }}$. This adds another free parameter to our model as $\alpha_{1}$ has been replaced by $\alpha_{1}+\alpha_{2} z$. However, 5 free parameters is still a small number considering this is a 3-dimensional model: $\rho(L, z)$. The redshift distribution is again specified by a two-tailed gaussian, as in model B. The 2 -D KS test was applied to the best-fits to this model and gave $P_{\mathrm{KS}}=0.4-0.7$ for all the cosmological models. MonteCarlo simulations using the model G RLF give $L-z$ planes indistinguishable from the true data. In addition, this model also fits the source counts at low fluxes well, giving a quasar fraction at $0.1 \mathrm{Jy}$ of $\approx 30 \%$. Similarly, we tested a variant 

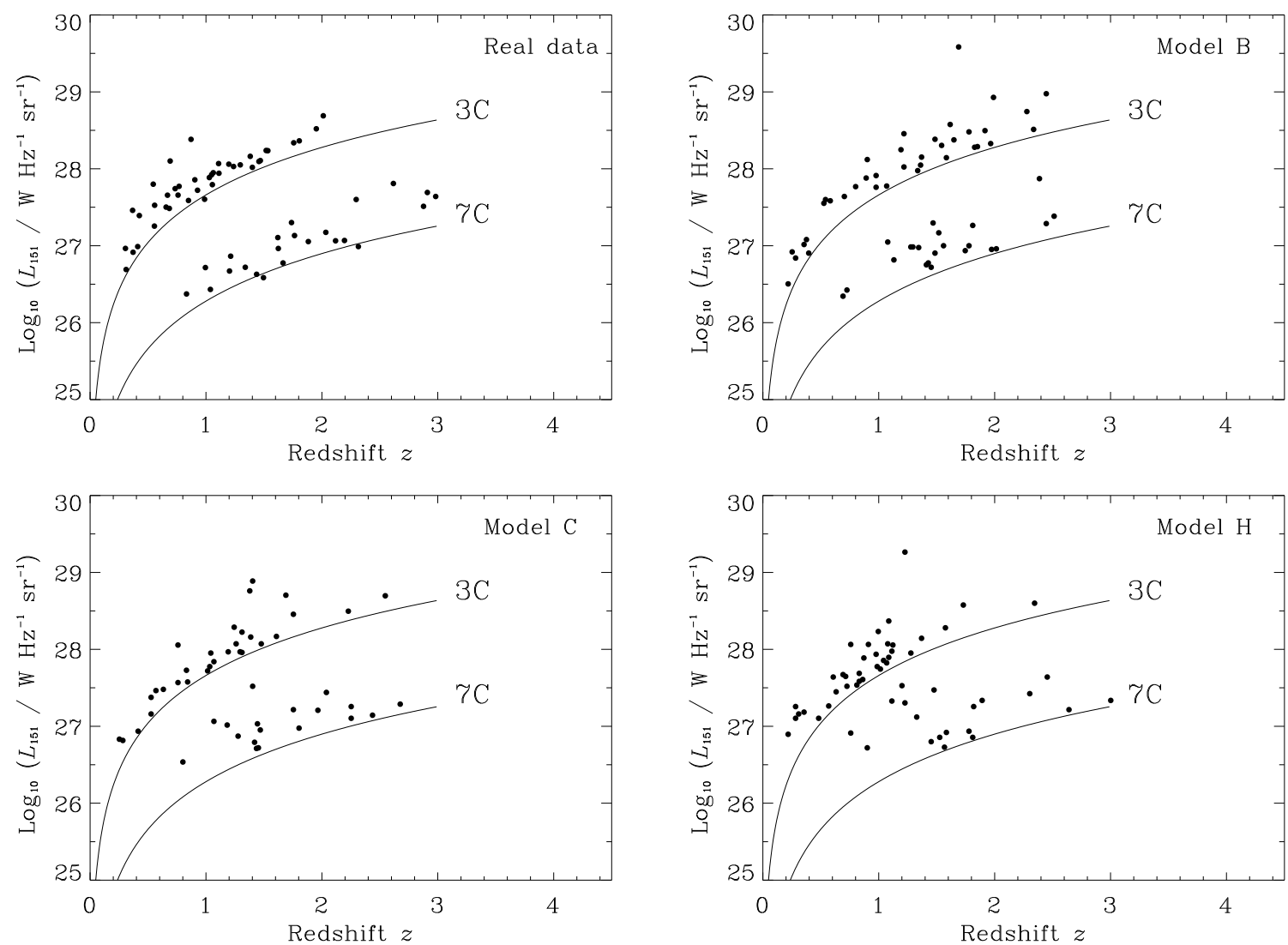

Figure 4. The plot at top-left shows the $L_{151}-z$ plane for the 3 CRR and $7 \mathrm{C}$ quasar samples. The other 3 plots show Monte-Carlo simulations of the $L_{151}-z$ plane for samples with the same flux limits and sky areas as the 3CRR and 7C samples. These simulations were generated using the best-fit RLF models B, C and H. All plots are for cosmology I.

of model $\mathrm{G}$ with a one-tailed gaussian redshift distribution (i.e. no decline in redshift for $z>z_{\circ}$ ). This model $(\mathrm{H})$ again fits well with KS probabilities in the range $0.4-0.9$ and good Monte-Carlo simulations and source counts fits. The likelihoods for model $\mathrm{H}$ are slightly higher than for model G.

Comparing the relative likelihoods for models $\mathrm{C}$ and $\mathrm{H}$, we find that for cosmology I model $\mathrm{H}$ has a 10 times greater likelihood than C, whereas for cosmologies II and III the likelihoods are comparable. Since $\mathrm{H}$ is a 5 parameter model, whereas $\mathrm{C}$ has only 4 parameters, we should only accept $\mathrm{H}$ as a best-fitting model if it can be shown that it is a significantly better fit than $\mathrm{C}$. To do this we need the ratio of the posterior probabilities for each model confronted with the data. The required ratio can be fairly approximated by the ratio of the likelihoods multiplied by an 'Ockham factor' $(<1)$ which penalises the extra latitude afforded to model $\mathrm{H}$ by an extra free parameter (e.g. Sivia 1996). For cosmologies II and III there is clearly no motivation to prefer model $\mathrm{H}$ (since their likelihood ratios are about 1 ). For cosmology I the ratio of the posterior probabilities is at most 10 , so although this hints that model $\mathrm{H}$ is to be preferred, uncertainty in the value of the Ockham factor ensures this remains a hint rather than a firm conclusion. The simulated $L_{151}-z$ data and source counts plots for both models are similar and fit the data well. Note that there are no 3CRR RLQs with $z>2.02$, despite the fact that the highest redshift RLQ,
$3 \mathrm{C} 9$, is luminous enough to be seen in the 3CRR sample at $z>3$ and $3 \mathrm{C} 191$ at $z \approx 2.7$. In contrast, there are nine 7C RLQs in the range $2<z<3$. This difference is reproduced slightly better by model $\mathrm{H}$ than model $\mathrm{C}$, due to the steepening of the RLF at high- $z$. An additional check on the validity of introducing an extra parameter comes from the size of its error. For model H, the extra parameter has the value $\alpha_{2}=0.46_{-0.29}^{+0.19}$. These errors are approximately equivalent to $1 \sigma$ errors, so for the additional term to become zero (and hence revert to a 4 parameter model) would only be a $1.6 \sigma$ result. Therefore, we conclude that we do not have sufficient evidence for the 5 parameter models to be considered a better fit than the 4 parameter models and hence model $\mathrm{C}$ remains our preferred model until further data suggest otherwise.

\subsection{Results}

The RLF models $\mathrm{C}$ and $\mathrm{H}$ are plotted in Figure $\mathrm{A}$ for $z=0.5$, 1, 2 and 3 (for cosmology I), along with the binned RLF of Section 4.1. Although at first glance, the binned and model RLFs appear quite different, these apparent differences are easily explained by the fact that the mean redshift varies from bin to bin, as discussed in Section 4.1. The fairly flat slope of the low- $z$ binned RLF is due to the mean redshift increasing from 0.5 to 1.0 as luminosity increases. This can clearly be seen by comparing the binned points with the 
model lines for $z=0.5$ and $z=1.0$. At high redshift $(1.2<$ $z<3.0$ ), both model $\mathrm{C}$ and $\mathrm{H}$ lines slightly underestimate the number density in several of the bins. This may simply be a consequence of small number statistics, because the largest deviation is only $\approx 2 \sigma$. The small sample size used here means that the RLF is only well-constrained in regions of the $L_{151}-z$ plane populated by $3 \mathrm{CRR}$ and $7 \mathrm{C}$ sources and extrapolations to other ranges of luminosity and redshift should be regarded with caution.

It is however, useful to consider extrapolation of the RLF to lower radio luminosities than those found in this sample. It has long been known that the radio luminosity distribution of quasars is bi-modal, with the separation into radio-quiet and radio-loud quasars occurring at $\log _{10}\left(L_{151}\right)$ $\sim 25.5$ (c.f. Kellermann et al. 1989). The $6 \mathrm{C}$ and $7 \mathrm{C}$ source counts show that a break in the power-law is required at $\log _{10}\left(L_{151}\right) \approx 27$, although we cannot constrain the slope of the RLQ luminosity function on the low luminosity side of this break or the exact luminosity of the break. The paucity of quasars (only 2) in the combined $3 \mathrm{CRR} / 7 \mathrm{C}$ sample with $\log _{10}\left(L_{151}\right) \leq 26.5$ is consistent with a break in the RLF near this luminosity. It should be noted however that 7 of the BLRGs in 3CRR \& $7 \mathrm{C}$ have $\log _{10}\left(L_{151}\right)<26.5$. In fact, due to the radio-optical correlation to be discussed in Section 5, BLRGs often have low radio luminosities and may contribute significantly to the quasar RLF at these radio luminosities. However, even including low optical luminosity quasars (BLRGs), there must still be a break in the RLQ RLF to be consistent with the $6 \mathrm{C} / 7 \mathrm{C}$ source counts.

The $6 \mathrm{C}$ complete sample of Eales $(1985 \mathrm{a}, \mathrm{b})$ was selected at $151 \mathrm{MHz}$ with flux limits $2.00 \leq S_{151} \leq 3.93$ Jy and covers $0.102 \mathrm{sr}$ (using the revised selection criteria from Blundell et al. 1998a). Hence it lies in between the $3 \mathrm{CRR}$ and $7 \mathrm{C}$ samples in the $L_{151}-z$ plane and can be used to check our RLF models. It contains only seven quasars (Eales 1985b; unpublished data), none of which are promoted into the sample by their core fluxes (Blundell et al. 1998a; unpublished data) . Integrating our model C RLF (for cosmology I) over the $6 \mathrm{C}$ flux limits and sky area, we find that there should be, on average, 16 quasars in the sample. Assuming Poisson statistics, $p(\leq 7)=0.01$ given mean $\mu=16$. The result is similar for model $\mathrm{H}$ with 15 quasars predicted in the $6 \mathrm{C}$ sample. It is therefore just plausible that the low quasar fraction in this sample (as commented on by Eales) is simply a statistical fluke.

The radio-loud quasar RLF derived here has some remarkably similar features to the OLF and XLF. The increase in $\log _{10} \rho(z)$ from $z \approx 0.5$ to $z \approx 2$ for model $\mathrm{C}$ is $\approx 1.5$, compared to $\approx 1.8$ for medium luminosity quasars in the XLF (Boyle et al. 1994) and $\approx 1.5$ for medium luminosity quasars in the OLF (Goldschmidt \& Miller 1998). Boyle et al. (1994) fit the quasar XLF with a redshift distribution which peaks at $z=1.6$ and then remains constant at higher redshifts, in accordance with our best-fitting RLF models. Of course, a decline in the OLF beyond $z \approx 3$ is now established (e.g. Warren, Hewett \& Osmer 1994; Schmidt, Schneider \& Gunn 1995), although these optically-selected samples could be biased if there is significant dust at high redshift. Flat-spectrum radio quasars also show decreasing number densities beyond $z \approx 3$ (Shaver et al. 1998), a result which is insensitive to the effects of dust. The high luminosity part of the OLF is known to steepen at high redshift
(Goldschmidt \& Miller 1998) and we find weak evidence that this may be occurring also in the RLF (e.g. model H). These similarities are important to pursue further because radio sources are known to be short-lived compared to the Hubble time (typically $10^{7} \mathrm{yr}$; Scheuer 1995), so the evolution must really be telling us about the evolution of the birth-rate (see also Blundell et al. 1998a), and by analogy the same for the OLF.

\section{THE RADIO-OPTICAL CORRELATION}

\subsection{Absolute $B$ magnitude calculation}

$K$-corrections in the $B$-band for high redshift objects can be very large and uncertain. The multi-colour photometry presented here avoids this potential source of error, because it allows the luminosity of an object in the rest-frame $B$ band to be calculated. For example, at $z \sim 3$ rest-frame $B$-band emission is observed at $1.8 \mu \mathrm{m}$ (close to the near-IR $K$-band). For sources at $z>0.5$, emitted $B$-band radiation is observed between the $R$ and $K$ bands. Therefore, for all 7C quasars a power-law continuum was fitted between the $R$ and $K$ magnitudes and a rest-frame $B$-band luminosity calculated. This was then converted to an absolute magnitude, $M_{B}$. The errors on these values are typically $\pm 0.2 \mathrm{mag}$; the main source of error being the $B$ and $R$ magnitudes from the APM catalogue. Paper VIII shows that this method provides a good estimate of $M_{B}$, even in quasars reddened by $A_{\mathrm{V}} \approx 1$.

For the 3CRR quasars, complete $K$-band photometry has not been published, so this approach was not possible. However, the maximum redshift in $3 \mathrm{CRR}$ is $\sim 2$, so the $K$ correction terms are not so large. For the $3 \mathrm{CRR}$ quasars a power-law optical continuum was calculated using published $V$ and $R$ magnitudes or $V$ and $B$ magnitudes, where available. For the few sources with only a published $V$ magnitude, an optical continuum slope of $\alpha_{\mathrm{opt}}=0.5$ was assumed. This slope is equivalent to $B-K \approx 3$ and is typical of unreddened quasars (Francis 1996). The calculated absolute magnitudes are listed in Table B1 in Appendix B. $K$-magnitudes were used (where available) in the calculation of the absolute magnitudes of $3 \mathrm{CRR}$ radio galaxies referred to in Section 2 .

\subsection{Results}

All the numerical results in this Section are presented for cosmology I. None of the conclusions differ significantly for either cosmology II or III. In Figure 5, we plot the calculated absolute magnitudes of the quasars and broad-line radio galaxies against redshift. The horizontal line is the dividing line in absolute magnitude between quasars and BLRGs from Section 3. The 5 CJSs are considered separately from the SSQs in this section because of possible orientation biases in their optical luminosities (e.g. Browne \& Wright 1985). The BLRGs are also excluded because they are probably not a complete sample. There is a very weak correlation between $M_{B}$ and $z$ for the SSQs (in the sense that the quasars increase in luminosity at greater $z$ ). Using Spearman's method of rank correlation, the significance of the correlation between $M_{B}$ and $z$ is $88 \%$. A radio-optical correlation is implicit in Figure 5 in the redshift range 


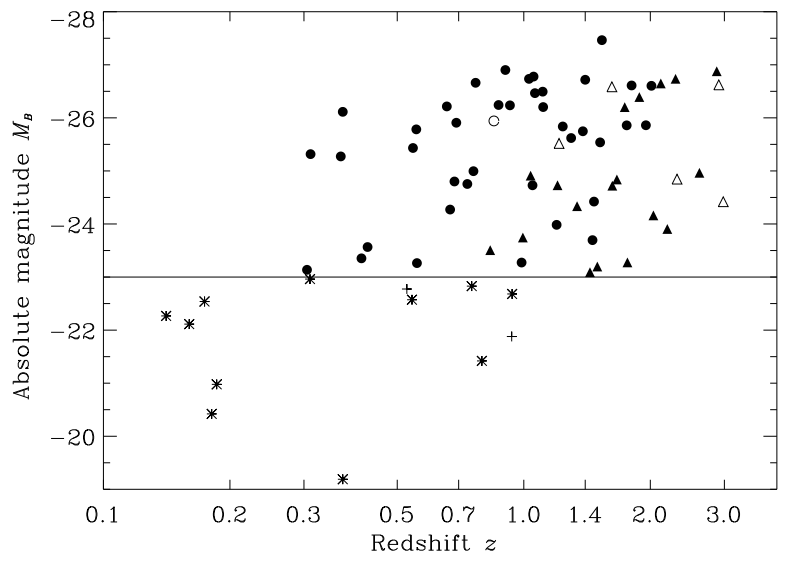

Figure 5. Absolute $B$ magnitude against redshift for the 3CRR and $7 \mathrm{C}$ quasar samples. The plotting symbols are the same as those used in Figure 1. BLRGs with $z \geq 0.1$ are also plotted here (asterisks - 3CRR, plus signs - 7C). This plot is for cosmology I.

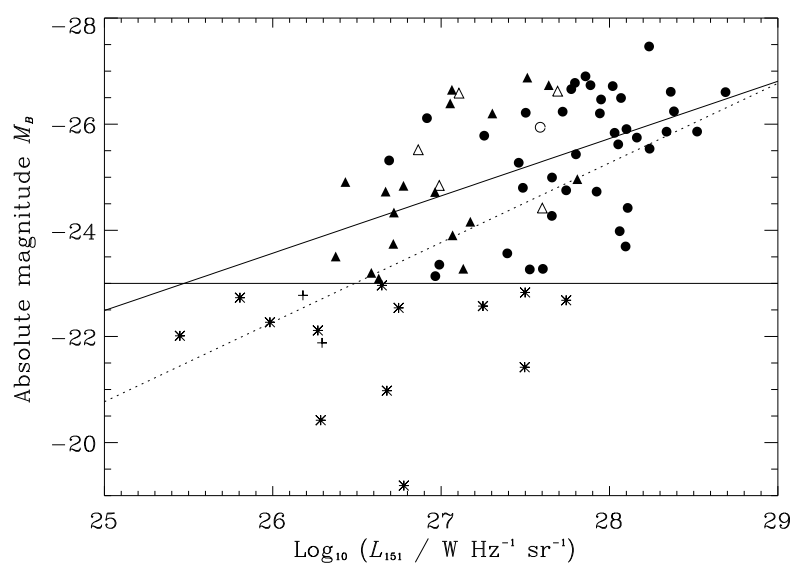

Figure 6. Absolute $\mathrm{B}$ magnitude against low-frequency radio luminosity for the $3 \mathrm{CRR}$ and $7 \mathrm{C}$ quasar samples. The plotting symbols are the same as those used in Figure 5. Plotted also is the best-fit straight line to the SSQs only, as described in the text (solid line). The dashed line is the radio-optical correlation for SSQs of Serjeant et al. (1998) transformed to cosmology I and $H_{\circ}=50 \mathrm{~km} \mathrm{~s}^{-1} \mathrm{Mpc}^{-1}$. This plot is for cosmology I.

$(1<z<2)$ where there is the most overlap between the $3 \mathrm{CRR}$ and $7 \mathrm{C}$ samples. It can clearly be seen that the $3 \mathrm{CRR}$ quasars (which have radio luminosities $\sim 25$ times greater than $7 \mathrm{C}$ quasars at the same $z$ ) are systematically brighter than those in $7 \mathrm{C}$. In this redshift range, the mean absolute magnitude, $\left\langle M_{B}\right\rangle$, for 3CRR SSQs is -25.8 with standard error 0.2 mag. The 7C SSQs have $\left\langle M_{B}\right\rangle=-24.6 \pm 0.3 \mathrm{mag}$, so are significantly fainter by about one magnitude. Note that the BLRGs appear to be more tightly correlated than the quasars on this plot. This is most likely due to the fact that all but 2 BLRGs are from the 3CRR sample, so there is a tight $L_{151}-z$ correlation.

Figure 6 shows the correlation between radio and optical luminosities for the combined 3CRR and 7C SSQ sam- ples. It can clearly be seen that there is a much stronger correlation than in Figure 5 . The significance of this correlation is $>99.9 \%$. This correlation does not appear to be caused by a correlation between $M_{B}$ and $z$ (and a $L_{151}-z$ correlation), because the statistical significance is very much greater. Also plotted on Figure 6 is the best-fit straight line to the 3CRR/7C SSQs (calculated by minimising the sum of the squares of the residuals). This line has a slope of $-2.5 \times(0.4 \pm 0.1)$, i.e. $L_{\mathrm{opt}} \propto L_{\mathrm{rad}}^{0.4}$. The dispersion about our straight-line fit is 1.0 magnitude. This is in fairly good agreement with Serjeant et al. (1998) who found a slightly steeper slope of $-2.5 \times(0.6 \pm 0.1)$, which is also plotted in Fig. 6 for comparison. One possible reason for our finding a flatter slope than Serjeant et al. follows from their lack of a limiting absolute magnitude in their definition of a quasar. Their sample contains quasars with $M_{B}>-23$ (which we would classify as BLRGs). These objects mostly have low radio luminosities, therefore increasing the slope of the correlation. This is evident in Fig. 6. There may be other systematic differences between the RLQs in the Serjeant et al. MAQS sample and those in the $7 \mathrm{C}$ sample. For example, the MAQS sample is selected at $408 \mathrm{MHz}$ leading to a higher proportion of compact steep-spectrum radio sources. We will consider these points in more detail in a future paper.

To investigate whether the radio-optical correlation could be due to a $M_{B}-z$ correlation, the Spearman partial rank correlation coefficient was used. This assesses the statistical significance of correlations between two variables in the presence of a third (Macklin 1982). For the correlation between $M_{B}$ and $z$ (independent of $L_{151}$ ), $r_{M_{B} z, L_{151}}=$ -0.13 with significance $D_{M_{B} z, L_{151}}=-1.0$. The significance is equivalent to the deviation from a unit variance normal distribution if there is no correlation present. Hence the correlation between $M_{B}$ and $z$ is marginally significant. Now we consider the correlation between $M_{B}$ and $L_{151}$ (independent of $z$ ). We find $r_{M_{B} L_{151}, z}=-0.43$ and $D_{M_{B} L_{151}, z}=-3.6$. Hence we can be sure that the obvious radio-optical correlation of Figure 6 is not caused by the $M_{B}-z$ correlation which plagues single flux-limited samples. The high radio luminosity but low optical luminosity (but brighter than $M_{B}=-23$ ) quasar region of Figure 6 is genuinely devoid of quasars. There are no selection effects which would cause a lack of quasars in this region.

Whenever a correlation such as this is presented, it is essential that all possible sources of error, both random and systematic, are considered. This is particularly important when combining samples with different selection criteria. The 3CRR and 7C SSQ samples are however, selected with very similar criteria (apart from their different flux limits). The only difference is their slightly different selection frequencies; 3CRR is selected at $178 \mathrm{MHz}$ and $7 \mathrm{C}$ at $151 \mathrm{MHz}$. Both are optically complete, because all the sources in the 3CRR sample and the 7C Redshift Survey have been identified. Hence we have eliminated one of the previously important possible sources of error, that of 'missing' quasars due to an optical magnitude limit.

There may be systematic errors between the two samples because the optical data available for them is inhomogeneous. The right side of Figure 6 is composed mostly of 3CRR objects and the left side mostly $7 \mathrm{C}$, so could this explain the observed correlation? The absolute magnitudes of 
the 7C quasars were calculated using multi-colour photometry, so are likely to be good estimates of the true values. Errors in $M_{B}$ due to reddening are not expected to be crucial here, because $R$ and $K$ magnitudes were predominantly used. Intrinsic reddening can be important when using $B$ magnitudes for high redshift objects, because the observed radiation was emitted in the UV. For the 3 CRR quasars, $M_{B}$ was calculated using optical photometry. Hence it is possible here that some quasars may be significantly reddened and this is a possible source of error. In addition, the magnitudes listed in LRL have not been corrected for galactic extinction. However, both these effects would tend to cause our calculated 3CRR $M_{B}$ values to be fainter than their true values. This cannot explain the observed radio-optical correlation, because the 3CRR quasars are systematically brighter than those in 7C. As noted above, the mean absolute magnitude of 3CRR SSQs is about 1 magnitude greater than the mean for $7 \mathrm{C}$ SSQs in the same redshift range. Hence the $3 \mathrm{CRR}$ photometry must be systematically in error by this amount for the correlation to disappear. It seems unlikely that this could be the case. The numerical results presented in this section are virtually independent of the choice of cosmology.

The data we have here is not detailed enough for us to explore the nature of the radio-optical correlation in quasars. For a discussion of possible physical causes of this effect, we refer the reader to Serjeant et al. (1998) and Paper III. Note that the CJSs tend to occupy positions on the optically bright side of the correlation. This could be explained by orientation-dependent optical emission, however the small number (6) of CJSs in the combined sample prevents us from drawing any firm conclusions.

\section{COMPLETENESS OF OTHER SAMPLES OF SSQS}

Using the quasar radio luminosity function derived in the previous section (model C), artificial RLQ samples were generated with a Monte-Carlo simulation for a range of $S_{151}$ flux limits from 0.1 to $20 \mathrm{Jy}$. Each sample contains approximately $5 \times 10^{4}$ sources, each defined by $L_{151}$ and $z$. The straightline fit to the radio-optical correlation for SSQs derived in Section 5 defines a characteristic value of $M_{B}$ for each value of $L_{151}$. The scatter about this straight-line was assumed to be independent of radio luminosity and a gaussian scatter of 1.0 magnitudes introduced into the $M_{B}$ calculation. For each source, $M_{B}$ and $z$ were then used to calculate its apparent $B$ magnitude (assuming $\alpha_{\mathrm{opt}}=0.5$ ). Quasars with $M_{B}>-23$ (BLRGs) were excluded from the simulated samples. The fraction of quasars brighter than the limiting $B$ magnitude $B_{\text {lim }}$ was then calculated for each sample as a function of $B_{\lim }$.

Figure 7 shows contours of the percentage of SSQs expected to be detected in surveys as a function of their optical and radio flux limits (for $\Omega_{M}=1, \Omega_{\Lambda}=0$ ). The immediate conclusion to be drawn here is that as surveys go to fainter radio fluxes, they must also go deeper optically, otherwise a significant fraction of quasars are missed. For $\Omega_{M}=0$, $\Omega_{\Lambda}=0$, the contours have steeper slopes across the diagram, such that they diverge from the $\Omega_{M}=1, \Omega_{\Lambda}=0$ contours at faint radio fluxes to be 0.5 magnitudes in $B$ fainter at $S_{151}=0.1 \mathrm{Jy}$. A similar behaviour is seen for $\Omega_{M}=0.1$,
$\Omega_{\Lambda}=0.9$. It must be remembered that these contours have been calculated assuming models for the radio-optical correlation and the quasar $\mathrm{RLF}$ from the combined $3 \mathrm{CRR} / 7 \mathrm{C}$ sample. Therefore in regions not probed by these samples, it is an extrapolation of the models. The area with the largest uncertainty is therefore $S_{151}<0.5 \mathrm{Jy}$. At $S_{151}=0.1 \mathrm{Jy}$, typical errors on the contours may be as large as \pm 1.0 in limiting $B$ magnitude.

According to Figure 7, $99 \%$ of the 3CRR SSQ sample has $B<20.5$ (i.e. brighter than the POSS limit). This is in good agreement with the fact that all of them are detected on the POSS plates. Our calculation predicts that 13 out of the $187 \mathrm{C}$ steep-spectrum quasars have $B<20.5$, compared with the actual number which is 11 . Note that the completeness percentages calculated here are strictly upper limits, because they do not take fully into account the faintness of reddened quasars. The completeness contours derived here only apply to low-frequency selected samples, e.g. $151 \mathrm{MHz}$. This is because high-frequency samples contain a large fraction of FSQs which are typically brighter than the mean SSQ radio-optical correlation (Wills \& Lynds, 1978; Browne \& Wright, 1985). Therefore FSQs are more likely to be brighter than the optical magnitude limits of samples.

We can use Figure 7 to estimate the completeness of various low-frequency radio selected quasar samples. This allows us to investigate possible bias in the sample properties due to selection effects. The large 7C quasar sample of Riley et al. (1998) has a faint radio flux limit of $S_{151}>0.1$ Jy and a bright optical limit of $R<20$. This sample is probably only approximately $40 \%$ complete. There are other new radio quasar samples which use POSS-I $(R \approx 20)$ identifications. The B3-VLA quasar sample (Vigotti et al. 1997) with a flux limit $S_{408} \geq 0.1 \mathrm{Jy}$ is estimated to be $55 \%$ complete from our plot (c.f. their estimate based on optical magnitude histograms of $70 \%$ ). For samples with $S_{151} \lesssim 1 \mathrm{Jy}$, one must use optical data significantly deeper than the POSS-I limit in order to avoid severe incompleteness in these samples.

The MAQS sample of Serjeant et al. (1998) is selected by $S_{408}>0.95$ Jy and $B<22.5$. We estimate that this sample is $99 \%$ complete, due to its faint optical limit (and its relatively bright radio limit). However, the higher selection frequency of $408 \mathrm{MHz}$ samples rest-frame $>1 \mathrm{GHz}$ emission for $z>1.5$ quasars. At these rest-frame frequencies cores and jets often dominate over extended emission, so the extended radio luminosity is lower and hence the quasars will be lower down the radio-optical correlation. It is possible however that a beamed optical-UV component, as discussed in the previous paragraph, may compensate in these objects. Therefore we conclude that the MAQS sample is likely to have excluded very few quasars due to its optical magnitude limit. A comparison of our RLF models with the MAQS sample will appear elsewhere (Serjeant et al. in preparation).

\section{CONCLUSIONS AND SUMMARY}

The sample of $7 \mathrm{C}$ quasars presented here is complete because every source in the $7 \mathrm{C}$ Redshift Survey has been identified. Therefore we do not suffer from colour biases excluding red and/or faint quasars. However, if orientation-based unified schemes (e.g. Antonucci 1993) are correct, then powerful radio galaxies are reddened quasars. For intermediate values 


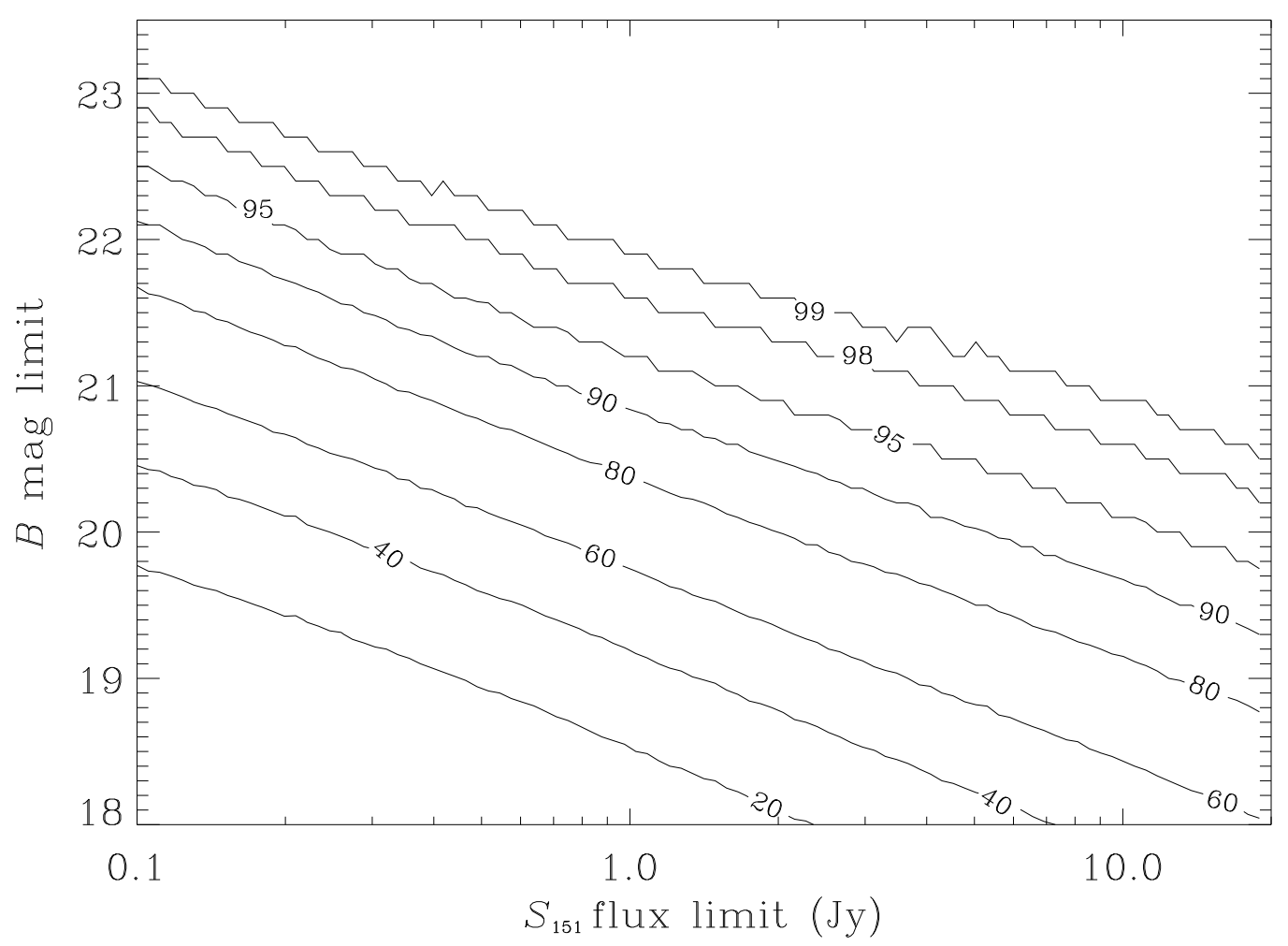

Figure 7. Percentage of quasars detected in surveys as a function of their optical and radio flux limits.This plot is for cosmology I.

of reddening $\left(\mathrm{A}_{V} \approx 2\right)$, different observers may classify objects as either radio galaxies or quasars. These issues will be investigated further in Paper VIII. The main conclusions to be drawn from this paper are:

- The radio luminosity function of radio-loud quasars has been derived. The best-fitting model is a single power-law with slope $\alpha_{1}=1.9 \pm 0.1$. We find that there must be a break in the RLQ RLF at $\log _{10}\left(L_{151} / \mathrm{W} \mathrm{Hz}^{-1} \mathrm{sr}^{-1}\right) \lesssim 27$, in order for the models to be consistent with the $7 \mathrm{C}$ and $6 \mathrm{C}$ source counts. The $z$-dependence of the RLF follows a one-tailed gaussian which peaks at $z=1.7 \pm 0.2$ and remains constant at higher redshifts. Models with or without a redshift cut-off beyond $z \approx 2$ fit almost equally well. We find marginal evidence that the RLF slope steepens as redshift increases. This form for the evolving RLF is very similar to the OLF determined from optically-selected samples (e.g. Goldschmidt \& Miller 1998).

- We have confirmed the existence of a significant correlation between the low-frequency radio and optical luminosities of SSQs, as reported in Serjeant et al. (1998). This correlation is not due to correlations with redshift or sample selection effects.

- Using the derived radio-loud quasar RLF and the observed radio-optical correlation, we have been able to estimate the fraction of quasars identified in low-frequency radio-selected samples with various magnitude limits. It is found that samples with faint radio flux limits must also go much deeper in the optical than the POSS-I plates $(R \approx 20)$, or there will be serious incompleteness in the samples. This would introduce many biases into the analysis of such samples, because only the optically brightest quasars would be included.

\section{ACKNOWLEDGEMENTS}

Special thanks to Steve Eales, Gary Hill, Julia Riley and David Rossitter for help with various aspects of the $7 \mathrm{C}$ Redshift Survey. We warmly thank the referee, Pippa Goldschmidt, for her constructive comments. We would like to thank Andrew Bunker and Isobel Hook for obtaining a spectrum of $5 \mathrm{C} 7.95$ for us and Richard Saunders for help with the spectroscopy of 5C6.95. We thank the staff at the WHT and UKIRT for technical support. The United Kingdom Infrared Telescope is operated by the Joint Astronomy Centre on behalf of the U.K. Particle Physics and Astronomy Research Council. We acknowledge the UKIRT Service Programme for some of the $K$-band imaging. The William Herschel Telescope is operated on the island of La Palma by the Isaac Newton Group in the Spanish Observatorio del Roque de los Muchachos of the Instituto de Astrofisica de Canarias. This research has made use of the NASA/IPAC Extra-galactic Database, which is operated by the Jet Propulsion Laboratory, Caltech, under contract with the National Aeronautics and Space Administration. CJW thanks PPARC for receipt of a studentship. 


\section{REFERENCES}

Antonucci R.R.J., 1984, ApJ, 278, 499

Antonucci R.R.J., 1993, ARAA, 31, 473

Avni Y., Bahcall J.N., 1980, ApJ, 235, 694

Baker J.C., 1997, MNRAS, 286, 23

Barthel P.D., Tytler D.R., Thomson B., 1990, A\&AS, 82, 339

Baum S.A., Heckman T.M., 1989, ApJ, 336, 702

Best P.N., Longair M.S., Röttgering H.J.A., 1998, MNRAS, 295, 549

Blundell K.M., Rawlings S., Willott C.J., 1998a, AJ, submitted (Paper IV)

Blundell K.M., Rawlings S., Willott C.J., Lacy M., Eales S.A., 1998b, in Observational Cosmology with the New Radio Surveys, eds. M.N. Bremer et al., 179, Kluwer

Blundell K.M., Rawlings S., Riley J.M., Willott C.J., Laing R.A., 1998c, to be submitted to New A (Paper I)

Boyle B.J., Fong R., Shanks T., Peterson B.A., 1987, MNRAS, 227,717

Boyle B.J., Shanks T., Peterson B.A., 1988, MNRAS, 235, 935

Boyle B.J., Shanks T., Georgantopoulos I., Stewart G.C., Griffiths R.E., 1994, MNRAS, 271, 639

Browne I.W.A., Wright A.E., 1985, MNRAS, 213, 97

Browne I.W.A., Murphy D., 1987, MNRAS, 226, 601

Cimatti A., Dey A., van Breugel W., Antonucci R., Spinrad H., 1996, ApJ, 465, 145

Della Ceca R., Maccacaro T., Gioia I.M., Wolter A., Stocke J.T., 1992, ApJ, 389, 491

Dunlop J.S., Peacock J.A., 1990, MNRAS, 247, 19

Eales S.A., 1985a, MNRAS, 217, 149

Eales S.A., 1985b, MNRAS, 217, 167

Eales S.A., Rawlings S., Law-Green J.D.B., Cotter G., Lacy M., 1997, MNRAS, 291, 593

Eracleous M., Halpern J.P., 1994, ApJS, 90, 1

Fanti C., Fanti R., Dallacasa D., Schilizzi R.T., Spencer R.E., Stanghellini C., 1995, A\&A, 302, 317

Francis P.J., 1996, PASA, 13, 212

Goldschmidt P., Miller L., 1998, MNRAS, 293, 107

Goodrich R.W., Cohen M.H., 1992, ApJ, 391, 623

Grandi S.A., Osterbrock D.E., 1978, ApJ, 220, 783

Gullixson C.A., Boeshaar P.C., Tyson J.A., Seitzer P., 1995, ApJS, 99, 281

Hales S.E.G., Baldwin J.E., Warner P.J., 1988, MNRAS, 234, 919

Hawkins M.R.S., Véron P., 1995, MNRAS, 275, 1102

Hewett P.C., Foltz C.B., Chaffee F.H., 1993, ApJ, 406, 43L

Hook I.M., McMahon R.G., Irwin M.J., Hazard C., 1996, MNRAS, 282, 1274

Irwin M.J., Maddox S.J., McMahon R.G., 1994, Spectrum: Newsletter of the Royal Observatories, 2, 14

Kellermann K.I., Sramek R., Schmidt M., Shaffer D.B., Green R., 1989, AJ, 98, 1195

Laing R.A., Riley J.M., Longair M.S., 1983, MNRAS, 204, 151 (LRL)

Laing R.A., Jenkins C.R., Wall J.V., Unger S.W., 1994, in Bicknell G., ed., Proceedings of the Stromlo Centenary Symposium: Physics of Active Galactic Nuclei. Springer-Verlag, Berlin, p.201

Lacy M., Rawlings S., 1996, MNRAS, 280, 888

Lacy M., Hill G.J., Kaiser M.E., Rawlings S., 1993, MNRAS, 263, 707

Lampton M., Margon B., Bowyer S., 1976, ApJ, 208, 177

Maccacaro T., Della Ceca R., Gioia I.M., Morris S.L., Stocke J.T.,Wolter A., 1991, ApJ, 374, 117

Macklin J.T., 1982, MNRAS, 199, 1119

Madau P., Ferguson H.C., Dickinson M.E., Giavalisco M., Steidel C.C., Fruchter A., 1996, MNRAS, 283, 1388

Marshall H.L., 1985, ApJ, 299, 109

Marshall H.L., Tanenbaum H., Huchra J.P., Braccesi A., Zitelli
V., 1983, ApJ 269, 42

McCarthy P.J., van Breugel W., Spinrad H., Djorgovski S., 1987, ApJ, 321, L29

McGilchrist M.M., Baldwin J.E., Riley J.M., Titterington D.J., Waldram E.M., Warner P.J., 1990, MNRAS, 246, 110

Miller L., Goldschmidt P., La Franca F., Cristiani S., 1993 in Observational Cosmology (ASP Conference Series No. 51), 614, eds. Chinarini G., Iovino A., Maccagini D.

O'Dea C.P., Baum S.A., Stanghellini C., 1991, ApJ, 380, 66

Ogle P.M., Cohen M.H., Miller J.S., Tran H.S., Fosbury R.A.E., Goodrich R.W., 1997, ApJ, 482L, 370

Peacock J.A., 1983, MNRAS, 202, 615

Pearson T.J., Kus A.J., 1978, MNRAS, 182, 273

Press W.H., Teukolsky S.A., Vetterling W.T., Flannery B.P., 1992, Numerical Recipes: The Art Of Scientific Computing. CUP, Cambridge.

Rawlings S., Saunders R., 1991, Nature, 349, 138

Rawlings S., Eales S.A., Warren S.J., 1990, MNRAS, 243, 14

Rawlings S., Lacy M., Sivia D.S., Eales S.A., 1995, MNRAS, 274, 428

Rawlings S., Blundell K.M., Lacy M., Willott C.J., Eales S.A., 1998, in Observational Cosmology with the New Radio Surveys, eds. M.N. Bremer et al., 171, Kluwer

Riley J.M., Rawlings S., McMahon R.G., Blundell K.M., Miller P., Lacy M., 1998, MNRAS, submitted

Roger R.S., Bridle A.H., Costain C.H., 1973, AJ, 78, 1030

Rossitter D., 1987, PhD thesis, University of Cambridge

Rowan-Robinson M., Jones M., Leech K., Vedi K., Hughes J., 1991, MNRAS, 249, 729

Savage B.D., Mathis J.S., 1979, ARAA, 17, 73

Scheuer P.A.G., 1987, in Superluminal Radio Sources, eds. J.A. Zensus and T.J. Pearson, p.331, Cambridge University Press

Scheuer P.A.G., 1995, MNRAS, 277, 331

Schmidt M., Schneider D.P., Gunn J.E., 1995, AJ, 110, 68

Seielstad G.A., Pearson T.J., Readhead A.C.S., 1983, PASP, 95, 842

Serjeant S., Rawlings S., Maddox S.J., Baker J.C., Clements D., Lacy M., Lilje P.B., 1998, MNRAS, 294, 494

Shaver P.A., Hook I.M., Jackson C., Wall J.V., Kellermann K.I., 1998, to appear in Highly Redshifted Radio Lines, eds. C. Carilli et al., (PASP: San Francisco), astro-ph/9801211

Sivia D.S., 1996, Data Analysis: a Bayesian Tutorial, OUP

Spinrad H., Smith H.E., 1976, ApJ, 206, 355

Spinrad H., Djorgovski S., Marr J., Aguilar L., 1985, PASP, 97, 932

Véron-Cetty M.P., Véron P., 1996, A catalogue of quasars and active nuclei (7th Edition), ESO Scientific Report No. 17, Garching bei Munchen

Vigotti M., Vettolani G., Merighi R., Lahulla J.F., Pedani M., 1997, AAS, 123, 219

Warren S.J., Hewett P.C., Osmer P.S., 1994, ApJ, 421, 412

Webster R.L., Francis P.J., Peterson B.A., Drinkwater M.J., Masci F.J., 1995, Nature, 375, 469.

Willott C.J., Rawlings S., Blundell K.M., Lacy M., 1998a, in Observational Cosmology with the New Radio Surveys, eds. M.N. Bremer et al., 209, Kluwer

Willott C.J., Rawlings S., Blundell K.M., Lacy M., 1998b, MNRAS submitted (Paper III)

Wills D., Lynds R., 1978, ApJS, 36, 317 


\section{APPENDIX A: OPTICAL SPECTRA}

In this appendix we present the optical spectra and emission line data for the 7C Redshift Survey quasars and broadlined radio galaxies. Figures A1 and A2 are the optical spectra of 5C6.264 and 5C6.288, respectively. These are the two quasars with no evidence of broad emission lines which will be discussed further in Paper VIII. Figure A3 displays the optical spectra for the other $247 \mathrm{C}$ quasars and broad-line radio galaxies. Prominent emission lines are labelled. Table A1 lists the properties of the emission lines measured from all these spectra. 


\begin{tabular}{|c|c|c|c|c|c|c|c|c|c|}
\hline name & $z$ & line & $\begin{array}{c}\lambda_{\text {rest }} \\
(\AA)\end{array}$ & $\begin{array}{c}\lambda_{\text {obs }} \\
(\AA)\end{array}$ & $\begin{array}{l}\text { FWHM } \\
(\AA)\end{array}$ & $\begin{array}{c}\text { flux } \\
\left(\mathrm{W} \mathrm{m}^{-2}\right)\end{array}$ & $\begin{array}{c}\mathrm{snr} \\
\sigma\end{array}$ & $\begin{array}{l}\mathrm{W}_{\lambda} \\
(\AA)\end{array}$ & notes \\
\hline $\begin{array}{l}5 \mathrm{C} 6.5 \\
7 \mathrm{C} \mathrm{B020414.4+332221}\end{array}$ & $\begin{array}{r}1.038 \\
\pm \\
.002\end{array}$ & $\begin{array}{l}\text { C III] } \\
\text { C II] } \\
\mathrm{Mg} \text { II }\end{array}$ & $\begin{array}{l}1909 \\
2326 \\
2799\end{array}$ & $\begin{array}{l}3887 \pm 15 \\
4743 \pm 10 \\
5704 \pm 2\end{array}$ & $\begin{array}{r}68 \pm 10 \\
58 \pm 12 \\
139 \pm 40\end{array}$ & $\begin{array}{l}1.2 \mathrm{e}-17 \\
2.6 \mathrm{e}-18 \\
1.3 \mathrm{e}-17\end{array}$ & $\begin{array}{r}5 \\
2.1 \\
4\end{array}$ & $\begin{array}{rr}71 & \pm 20 \\
19 & \pm \\
138 & \pm\end{array}$ & abs \\
\hline $\begin{array}{l}5 \mathrm{C} 6.8 \\
7 \mathrm{C} \mathrm{B020439.6+313752}\end{array}$ & $\begin{array}{r}1.213 \\
\pm \\
.002\end{array}$ & $\begin{array}{l}\text { C IV } \\
\mathrm{He} \text { II } \\
\mathrm{C} \text { III] } \\
\mathrm{Mg} \text { II } \\
{\left[\begin{array}{ll}\mathrm{O} & \mathrm{II}\end{array}\right]}\end{array}$ & $\begin{array}{l}1549 \\
1640 \\
1909 \\
2799 \\
3727\end{array}$ & $\begin{array}{l}3434 \pm \\
3626 \pm 3 \\
4213 \pm 5 \\
6196 \pm 5 \\
8247 \pm 5\end{array}$ & $\begin{array}{r}34 \pm 20 \\
23 \pm 6 \\
110 \pm 30 \\
101 \pm 20 \\
24 \pm 5\end{array}$ & $\begin{array}{l}1.6 \mathrm{e}-17 \\
3.4 \mathrm{e}-18 \\
1.4 \mathrm{e}-17 \\
1.0 \mathrm{e}-17 \\
1.0 \mathrm{e}-18\end{array}$ & $\begin{array}{r}2.8 \\
1.6 \\
5 \\
6 \\
1.3\end{array}$ & $\begin{array}{lr}65 \pm & 35 \\
16 \pm & 8 \\
81 \pm & 30 \\
71 \pm & 16 \\
23 \pm & 8\end{array}$ & $\begin{array}{l}\text { in noise } \\
\text { abs }\end{array}$ \\
\hline $\begin{array}{l}\text { 5C6.33 } \\
\text { 7C B020836.3+333419 }\end{array}$ & $\begin{array}{r}1.496 \\
\pm \\
.003\end{array}$ & $\begin{array}{l}\text { C IV } \\
\text { He II } \\
\text { C III] } \\
\text { Mg II }\end{array}$ & $\begin{array}{l}1549 \\
1640 \\
1909 \\
2799\end{array}$ & $\begin{array}{l}3855 \pm 10 \\
4098 \pm 10 \\
4752 \pm 20 \\
6998 \pm 20\end{array}$ & $\begin{array}{l}150 \pm 40 \\
110 \pm 30 \\
170 \pm 40 \\
209 \pm 50\end{array}$ & $\begin{array}{l}1.9 \mathrm{e}-18 \\
4.0 \mathrm{e}-19 \\
9.5 \mathrm{e}-19 \\
8.8 \mathrm{e}-19\end{array}$ & $\begin{array}{r}7 \\
2.3 \\
4 \\
4\end{array}$ & $\begin{array}{r}220 \pm 70 \\
53 \pm 17 \\
229 \pm 70 \\
200 \pm 60\end{array}$ & $\begin{array}{l}\text { abs } \\
\text { abs } \\
\text { abs }\end{array}$ \\
\hline $\begin{array}{l}5 \text { C6.34 } \\
7 \text { C B020838.5+312131 }\end{array}$ & $\begin{array}{r}2.118 \\
\pm \\
.002\end{array}$ & $\begin{array}{l}\text { Ly } \alpha / \text { N V } \\
\text { C IV } \\
\text { He II } \\
\text { C III] }\end{array}$ & $\begin{array}{l}1216 \\
1549 \\
1640 \\
1909\end{array}$ & $\begin{array}{l}3790 \pm 6 \\
4827 \pm 3 \\
5116 \pm 4 \\
5936 \pm 15\end{array}$ & $\begin{aligned} 47 & \pm 10 \\
76 & \pm 10 \\
42 & \pm 8 \\
146 & \pm 20\end{aligned}$ & $\begin{array}{l}5.3 \mathrm{e}-17 \\
1.5 \mathrm{e}-17 \\
6.1 \mathrm{e}-18 \\
8.1 \mathrm{e}-18\end{array}$ & $\begin{array}{r}35 \\
15 \\
6 \\
6\end{array}$ & $\begin{array}{r}198 \pm \\
71 \pm \\
38 \pm \\
\pm 10 \\
56 \pm 30\end{array}$ & $\begin{array}{l}\text { abs } \\
\text { abs } \\
\text { cr in line }\end{array}$ \\
\hline $\begin{array}{l}5 \mathrm{C} 6.39 \\
7 \mathrm{C} \mathrm{B020853.8+321446}\end{array}$ & $\begin{array}{r}1.437 \\
\pm \\
.003\end{array}$ & $\begin{array}{l}\text { Si IV / O IV } \\
\text { C IV } \\
\text { He II } \\
\text { C III] } \\
\text { C II] } \\
\text { Mg II } \\
{[\mathrm{Ne} \mathrm{V]}}\end{array}$ & $\begin{array}{l}1400 \\
1549 \\
1640 \\
1909 \\
2326 \\
2799 \\
3426\end{array}$ & $\begin{array}{l}3408 \pm 15 \\
3774 \pm 3 \\
3994 \pm 3 \\
4644 \pm 5 \\
5659 \pm 12 \\
6813 \pm 17 \\
8348 \pm 5\end{array}$ & $\begin{aligned} 57 & \pm 20 \\
65 & \pm 12 \\
17 & \pm 7 \\
89 & \pm 19 \\
45 & \pm 10 \\
146 & \pm 25 \\
50 & \pm 25\end{aligned}$ & $\begin{array}{l}8.9 \mathrm{e}-19 \\
6.1 \mathrm{e}-18 \\
3.5 \mathrm{e}-19 \\
1.2 \mathrm{e}-18 \\
3.6 \mathrm{e}-19 \\
1.9 \mathrm{e}-18 \\
1.8 \mathrm{e}-19\end{array}$ & $\begin{array}{r}7 \\
70 \\
6 \\
9 \\
5 \\
15 \\
4\end{array}$ & $\begin{aligned} 193 & \pm 120 \\
415 & \pm 80 \\
47 & \pm 40 \\
220 & \pm 35 \\
71 & \pm 28 \\
475 & \pm 110 \\
81 & \pm 46\end{aligned}$ & $\begin{array}{l}\text { poss. broad base } \\
\text { abs } \\
\text { abs and atm abs }\end{array}$ \\
\hline $\begin{array}{l}5 \mathrm{C} 6.95 \\
7 \mathrm{C} \mathrm{B021149.2+293640}\end{array}$ & $\begin{array}{r}2.877 \\
\pm \\
.007\end{array}$ & $\begin{array}{l}\text { Ly } \alpha \\
\text { N V } \\
\text { C IV } \\
\text { C III] }\end{array}$ & $\begin{array}{l}1216 \\
1240 \\
1549 \\
1909\end{array}$ & $\begin{array}{l}4726 \pm 13 \\
4812 \pm 3 \\
5986 \pm 20 \\
7396 \pm 25\end{array}$ & $\begin{aligned} 109 & \pm 9 \\
21 & \pm 4 \\
92 & \pm 11 \\
128 & \pm 24\end{aligned}$ & $\begin{array}{l}5.6 \mathrm{e}-18 \\
7.1 \mathrm{e}-19 \\
2.4 \mathrm{e}-18 \\
2.7 \mathrm{e}-18\end{array}$ & $\begin{array}{r}14 \\
1.8 \\
14 \\
14\end{array}$ & $\begin{array}{r}375 \pm 90 \\
60 \pm 12 \\
95 \pm 35 \\
117 \pm 40\end{array}$ & $\begin{array}{l}\text { abs } \\
\text { abs } \\
\text { abs }\end{array}$ \\
\hline $\begin{array}{l}5 \mathrm{C} 6.160 \\
7 \mathrm{C} \mathrm{B} 021444.8+332114\end{array}$ & $\begin{array}{r}1.624 \\
\pm \\
.002\end{array}$ & $\begin{array}{l}\text { C IV } \\
\mathrm{He} \text { II } \\
\mathrm{C} \text { III] } \\
\mathrm{Mg} \text { II }\end{array}$ & $\begin{array}{l}1549 \\
1640 \\
1909 \\
2799\end{array}$ & $\begin{array}{l}4065 \pm 5 \\
4304 \pm 5 \\
5004 \pm 7 \\
7351 \pm 20\end{array}$ & $\begin{aligned} 66 & \pm 8 \\
53 & \pm 12 \\
78 & \pm 20 \\
140 & \pm 60\end{aligned}$ & $\begin{array}{l}3.2 \mathrm{e}-17 \\
2.7 \mathrm{e}-18 \\
1.1 \mathrm{e}-17 \\
8.0 \mathrm{e}-18\end{array}$ & $\begin{array}{r}13 \\
1.7 \\
6 \\
1.5\end{array}$ & 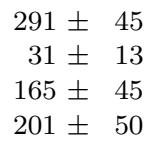 & $\begin{array}{l}\text { abs } \\
\text { abs } \\
\text { abs } \\
\text { abs }\end{array}$ \\
\hline $\begin{array}{l}5 \mathrm{C} 6.237 \\
7 \mathrm{C} \mathrm{B} 021749.9+322724\end{array}$ & $\begin{array}{r}1.620 \\
\pm \\
.003\end{array}$ & $\begin{array}{l}\text { C IV } \\
\mathrm{He} \text { II } \\
\mathrm{C} \text { III] } \\
\mathrm{Mg} \text { II }\end{array}$ & $\begin{array}{l}1549 \\
1640 \\
1909 \\
2799\end{array}$ & $\begin{array}{l}4058 \pm 15 \\
4280 \pm 20 \\
5007 \pm 15 \\
7332 \pm 8\end{array}$ & $\begin{aligned} 70 & \pm 20 \\
86 & \pm 25 \\
102 & \pm 28 \\
82 & \pm 24\end{aligned}$ & $\begin{array}{l}2.6 \mathrm{e}-17 \\
1.0 \mathrm{e}-17 \\
1.4 \mathrm{e}-17 \\
4.0 \mathrm{e}-18\end{array}$ & $\begin{array}{r}12 \\
4 \\
5 \\
2.6\end{array}$ & $\begin{array}{lr}78 \pm & 28 \\
35 \pm & 7 \\
52 & \pm \\
27 & \pm 10\end{array}$ & $\begin{array}{l}\text { abs \& cr in line } \\
\text { abs } \\
\text { abs \& cr in line }\end{array}$ \\
\hline $\begin{array}{l}5 \mathrm{C} 6.251 \\
7 \mathrm{C} \mathrm{B} 021850.4+340239\end{array}$ & $\begin{array}{r}1.665 \\
\pm \\
.002\end{array}$ & $\begin{array}{l}\text { Si IV / O IV } \\
\text { C IV } \\
\text { He II } \\
\text { C III] } \\
\text { C II] } \\
\text { [Ne IV] } \\
\text { Mg II }\end{array}$ & $\begin{array}{l}1400 \\
1549 \\
1640 \\
1909 \\
2326 \\
2424 \\
2799\end{array}$ & $\begin{array}{l}3726 \pm 8 \\
4107 \pm 12 \\
4376 \pm 25 \\
5060 \pm 20 \\
6199 \pm 6 \\
6463 \pm 4 \\
7410 \pm 40\end{array}$ & $\begin{aligned} 44 & \pm 8 \\
91 & \pm 10 \\
110 & \pm 60 \\
114 & \pm 40 \\
72 & \pm 28 \\
20 & \pm 4 \\
155 & \pm 25\end{aligned}$ & $\begin{array}{l}5.8 \mathrm{e}-18 \\
1.2 \mathrm{e}-17 \\
3.2 \mathrm{e}-18 \\
7.2 \mathrm{e}-18 \\
1.0 \mathrm{e}-18 \\
2.1 \mathrm{e}-19 \\
5.2 \mathrm{e}-18\end{array}$ & $\begin{array}{r}5 \\
8 \\
1.2 \\
3.4 \\
1.3 \\
1.1 \\
4\end{array}$ & $\begin{array}{rr}47 \pm & 18 \\
108 \pm & 15 \\
30 \pm & 15 \\
85 \pm & 24 \\
21 \pm & 9 \\
4 \pm & 3 \\
131 \pm & 50\end{array}$ & $\begin{array}{l}\text { abs } \\
\text { atm abs }\end{array}$ \\
\hline $\begin{array}{l}\text { 5C6.264 } \\
\text { 7C B021949.1+334341 }\end{array}$ & $\begin{array}{r}0.832 \\
\pm \\
.001\end{array}$ & {$[\mathrm{OII}]$} & 3727 & $6829 \pm 2$ & $23 \pm 2$ & $1.8 \mathrm{e}-19$ & 8 & $62 \pm 15$ & \\
\hline $\begin{array}{l}\text { 5C6.282 } \\
\text { 7C B022140.8+340609 }\end{array}$ & $\begin{array}{r}2.195 \\
\pm \\
.003\end{array}$ & $\begin{array}{l}\text { Ly } \alpha \\
\text { N V } \\
\text { C IV } \\
\text { He II } \\
\text { C III] }\end{array}$ & $\begin{array}{l}1216 \\
1240 \\
1549 \\
1640 \\
1909\end{array}$ & $\begin{array}{l}3902 \pm 10 \\
3965 \pm 5 \\
4950 \pm 20 \\
5235 \pm 4 \\
6063 \pm 30\end{array}$ & $\begin{aligned} 17 & \pm 5 \\
43 & \pm 12 \\
150 & \pm 40 \\
80 & \pm 32 \\
123 & \pm 40\end{aligned}$ & $\begin{array}{l}3.2 \mathrm{e}-19 \\
4.2 \mathrm{e}-19 \\
3.5 \mathrm{e}-19 \\
1.7 \mathrm{e}-19 \\
1.4 \mathrm{e}-19\end{array}$ & $\begin{array}{r}2.9 \\
4 \\
3.5 \\
2.9 \\
1.8\end{array}$ & 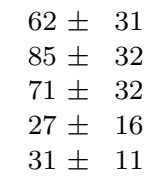 & $\begin{array}{l}\text { abs } \\
\text { abs } \\
\text { abs }\end{array}$ \\
\hline $\begin{array}{l}5 \mathrm{C} 6.286 \\
7 \mathrm{C} \mathrm{B} 022209.4+314550\end{array}$ & $\begin{array}{r}1.339 \\
\pm \\
.003\end{array}$ & $\begin{array}{l}\mathrm{C} \text { IV } \\
\mathrm{C} \text { III] } \\
\mathrm{Mg} \text { II }\end{array}$ & $\begin{array}{l}1549 \\
1909 \\
2799\end{array}$ & $\begin{array}{l}3625 \pm 7 \\
4451 \pm 10 \\
6563 \pm 20\end{array}$ & $\begin{aligned} 74 & \pm 6 \\
86 & \pm 15 \\
169 & \pm 25\end{aligned}$ & $\begin{array}{l}2.9 \mathrm{e}-17 \\
6.3 \mathrm{e}-18 \\
8.8 \mathrm{e}-18\end{array}$ & $\begin{array}{r}7 \\
2.6 \\
3.3\end{array}$ & $\begin{array}{l}400 \pm 100 \\
121 \pm 38 \\
460 \pm 100\end{array}$ & abs \\
\hline
\end{tabular}

Table A1. 


\begin{tabular}{|c|c|c|c|c|c|c|c|c|c|}
\hline name & $z$ & line & $\begin{array}{c}\lambda_{\text {rest }} \\
(\AA)\end{array}$ & $\begin{array}{c}\lambda_{\text {obs }} \\
(\AA)\end{array}$ & $\begin{array}{c}\text { FWHM } \\
(\AA)\end{array}$ & $\begin{array}{c}\text { flux } \\
\left(\mathrm{W} \mathrm{m}^{-2}\right)\end{array}$ & $\begin{array}{c}\mathrm{snr} \\
\sigma\end{array}$ & $\begin{array}{l}\mathrm{W}_{\lambda} \\
(\AA)\end{array}$ & notes \\
\hline $\begin{array}{l}5 \mathrm{C} 6.287 \\
7 \mathrm{C} \mathrm{B} 022220.0+313901\end{array}$ & $\begin{array}{r}2.296 \\
\pm \\
.002\end{array}$ & $\begin{array}{l}\text { Ly } \alpha / \text { N V } \\
\text { C IV } \\
\text { He II } \\
\text { C III] }\end{array}$ & $\begin{array}{l}1216 \\
1549 \\
1640 \\
1909\end{array}$ & $\begin{array}{l}4005 \pm 5 \\
5114 \pm 15 \\
5408 \pm 8 \\
6288 \pm 12\end{array}$ & $\begin{aligned} 88 & \pm 12 \\
101 & \pm 18 \\
35 & \pm 30 \\
80 & \pm 40\end{aligned}$ & $\begin{array}{l}8.5 \mathrm{e}-17 \\
3.5 \mathrm{e}-17 \\
8.5 \mathrm{e}-18 \\
3.7 \mathrm{e}-18\end{array}$ & $\begin{array}{r}15 \\
8 \\
1.4 \\
1.9\end{array}$ & $\begin{aligned} 340 & \pm 100 \\
192 & \pm \\
57 & \pm 35 \\
145 & \pm 25\end{aligned}$ & abs \\
\hline $\begin{array}{l}5 \mathrm{C} 6.288 \\
7 \mathrm{C} \mathrm{B} 022219.3+310527\end{array}$ & $\begin{array}{r}2.982 \\
\pm \\
.003\end{array}$ & $\begin{array}{l}\text { Ly } \alpha \\
\text { C IV } \\
\text { He II }\end{array}$ & $\begin{array}{l}1216 \\
1549 \\
1640\end{array}$ & $\begin{array}{l}4842 \pm 5 \\
6181 \pm 6 \\
6541 \pm 6\end{array}$ & $\begin{array}{l}23 \pm 3 \\
22 \pm 5 \\
24 \pm 6\end{array}$ & $\begin{array}{l}6.1 \mathrm{e}-19 \\
2.7 \mathrm{e}-20 \\
4.2 \mathrm{e}-20\end{array}$ & $\begin{array}{l}12 \\
1.4 \\
1.6\end{array}$ & $\begin{array}{r}240 \pm 100 \\
36 \pm 15 \\
34 \pm 12\end{array}$ & \\
\hline $\begin{array}{l}5 \mathrm{C} 6.291 \\
7 \mathrm{C} \mathrm{B} 022309.7+340801\end{array}$ & $\begin{array}{r}2.910 \\
\pm \\
.002\end{array}$ & $\begin{array}{l}\text { Ly } \alpha / \text { N V } \\
\text { C IV } \\
\text { He II } \\
\text { C III] }\end{array}$ & $\begin{array}{l}1216 \\
1549 \\
1640 \\
1909\end{array}$ & $\begin{array}{l}4752 \pm 1 \\
6022 \pm 2 \\
6417 \pm 2 \\
7465 \pm 2\end{array}$ & $\begin{array}{r}22 \pm 2 \\
25 \pm 5 \\
30 \pm 4 \\
145 \pm 25\end{array}$ & $\begin{array}{l}2.9 \mathrm{e}-18 \\
1.7 \mathrm{e}-19 \\
2.2 \mathrm{e}-19 \\
7.6 \mathrm{e}-19\end{array}$ & $\begin{array}{r}90 \\
12 \\
9 \\
17\end{array}$ & 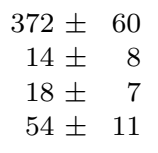 & $\begin{array}{l}\text { abs } \\
\text { abs } \\
\text { narrow core }\end{array}$ \\
\hline $\begin{array}{l}\text { 7C0808+2854 } \\
7 \mathrm{C} \mathrm{B} 080832.1+285402\end{array}$ & $\begin{array}{r}1.883 \\
\pm \\
.003\end{array}$ & $\begin{array}{l}\text { Ly } \alpha \\
\text { Si IV / O IV } \\
\text { C IV } \\
\text { C III] } \\
\text { Mg II }\end{array}$ & $\begin{array}{l}1216 \\
1400 \\
1549 \\
1909 \\
2799\end{array}$ & $\begin{array}{l}3518 \pm 12 \\
4020 \pm 10 \\
4466 \pm 9 \\
5485 \pm 10 \\
8070 \pm 4\end{array}$ & $\begin{array}{l}105 \pm 20 \\
117 \pm 25 \\
108 \pm 20 \\
130 \pm 25 \\
200 \pm 40\end{array}$ & $\begin{array}{l}5.8 \mathrm{e}-17 \\
5.2 \mathrm{e}-18 \\
1.9 \mathrm{e}-17 \\
7.0 \mathrm{e}-18 \\
2.1 \mathrm{e}-18\end{array}$ & $\begin{array}{r}50 \\
6 \\
15 \\
8 \\
3.3\end{array}$ & 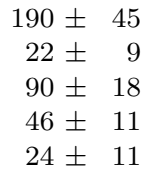 & $\begin{array}{l}\text { abs } \\
\text { abs } \\
\text { abs } \\
\text { abs }\end{array}$ \\
\hline $\begin{array}{l}5 \mathrm{C} 7.17 \\
7 \mathrm{C} \mathrm{B} 080956.4+270058\end{array}$ & $\begin{array}{r}0.936 \\
\pm \\
.001\end{array}$ & $\begin{array}{l}\mathrm{C} \text { III }] \\
\mathrm{Mg} \text { II } \\
{[\mathrm{Ne} \mathrm{V}]} \\
{[\mathrm{O} \quad \mathrm{II}]} \\
{[\mathrm{Ne} \mathrm{III}]}\end{array}$ & $\begin{array}{l}1909 \\
2799 \\
3346 \\
3727 \\
3869\end{array}$ & $\begin{array}{l}3695 \pm 15 \\
5407 \pm 15 \\
6479 \pm 2 \\
7217 \pm 3 \\
7488 \pm 2\end{array}$ & $\begin{array}{r}95 \pm 25 \\
202 \pm 60 \\
14 \pm \quad 4 \\
23 \pm 3 \\
16 \pm 3\end{array}$ & $\begin{array}{l}1.8 \mathrm{e}-18 \\
1.4 \mathrm{e}-18 \\
9.6 \mathrm{e}-20 \\
3.5 \mathrm{e}-19 \\
9.9 \mathrm{e}-20\end{array}$ & $\begin{array}{r}9 \\
8 \\
1.8 \\
9 \\
2.5\end{array}$ & $\begin{array}{r}302 \pm 140 \\
260 \pm 120 \\
27 \pm \quad 9 \\
76 \pm 18 \\
24 \pm \quad 7\end{array}$ & abs \\
\hline $\begin{array}{l}5 \mathrm{C} 7.70 \\
7 \mathrm{C} \mathrm{B} 081400.9+292731\end{array}$ & $\begin{array}{r}2.617 \\
\pm \\
.003\end{array}$ & $\begin{array}{l}\text { Ly } \alpha \\
\text { C IV } \\
\text { He II } \\
\text { C III] }\end{array}$ & $\begin{array}{l}1216 \\
1549 \\
1640 \\
1909\end{array}$ & $\begin{array}{l}4420 \pm 4 \\
5633 \pm 12 \\
5947 \pm 14 \\
6905 \pm 5\end{array}$ & $\begin{aligned} 40 & \pm 10 \\
100 & \pm 50 \\
140 & \pm 70 \\
100 & \pm 50\end{aligned}$ & $\begin{array}{l}3.9 \mathrm{e}-19 \\
1.9 \mathrm{e}-19 \\
1.8 \mathrm{e}-19 \\
4.2 \mathrm{e}-19\end{array}$ & $\begin{array}{r}8 \\
2.6 \\
2.2 \\
8\end{array}$ & $\begin{array}{l}88 \pm 37 \\
37 \pm 25 \\
37 \pm 25 \\
95 \pm 29\end{array}$ & $\begin{array}{l}\text { abs } \\
\text { abs } \\
\text { abs }\end{array}$ \\
\hline $\begin{array}{l}5 \mathrm{C} 7.85 \\
7 \mathrm{C} \mathrm{B} 081445.6+272601\end{array}$ & $\begin{array}{r}0.995 \\
\pm \\
.001\end{array}$ & $\begin{array}{l}\mathrm{C} \text { III }] \\
\mathrm{Mg} \text { II } \\
{[\mathrm{O} \mathrm{II}]} \\
{[\mathrm{Ne} \text { III }]}\end{array}$ & $\begin{array}{l}1909 \\
2799 \\
3727 \\
3869\end{array}$ & $\begin{array}{l}3799 \pm 7 \\
5602 \pm 12 \\
7436 \pm 3 \\
7718 \pm 5\end{array}$ & $\begin{aligned} 70 & \pm 30 \\
128 & \pm 20 \\
29 & \pm 3 \\
29 & \pm 3\end{aligned}$ & $\begin{array}{l}9.3 \mathrm{e}-19 \\
2.1 \mathrm{e}-18 \\
2.6 \mathrm{e}-19 \\
2.9 \mathrm{e}-19\end{array}$ & $\begin{array}{r}6 \\
10 \\
3.6 \\
3.8\end{array}$ & $\begin{array}{lr}43 \pm & 20 \\
77 \pm & 22 \\
11 \pm & 3 \\
14 \pm & 3\end{array}$ & $\begin{array}{l}\text { cr in line } \\
\text { abs }\end{array}$ \\
\hline $\begin{array}{l}5 \mathrm{C} 7.87 \\
7 \mathrm{C} \mathrm{B} 081503.9+245827\end{array}$ & $\begin{array}{r}1.764 \\
\pm \\
.002\end{array}$ & $\begin{array}{l}\text { Ly } \alpha \\
\text { C IV } \\
\text { He II } \\
\text { C III] } \\
\text { Mg II }\end{array}$ & $\begin{array}{l}1216 \\
1549 \\
1640 \\
1909 \\
2799\end{array}$ & $\begin{array}{l}3370 \pm 4 \\
4278 \pm 8 \\
4534 \pm 5 \\
5263 \pm 10 \\
7742 \pm 10\end{array}$ & $\begin{array}{l}28 \pm 16 \\
71 \pm 15 \\
69 \pm 23 \\
52 \pm 30 \\
91 \pm 30\end{array}$ & $\begin{array}{l}3.0 \mathrm{e}-18 \\
4.2 \mathrm{e}-18 \\
4.5 \mathrm{e}-19 \\
9.1 \mathrm{e}-19 \\
5.8 \mathrm{e}-19\end{array}$ & $\begin{array}{r}2.7 \\
30 \\
6 \\
9 \\
3.3\end{array}$ & 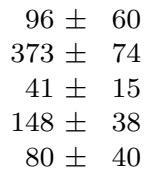 & $\begin{array}{l}\text { in noise } \\
\text { abs } \\
\text { in atm abs }\end{array}$ \\
\hline $\begin{array}{l}5 \mathrm{C} 7.95 \\
7 \mathrm{C} \mathrm{B} 081520.0+244506\end{array}$ & $\begin{array}{r}1.203 \\
\pm \\
.002\end{array}$ & $\begin{array}{l}\text { C IV } \\
\text { C III] } \\
\text { Mg II } \\
{\left[\begin{array}{ll}O \\
\text { II }]\end{array}\right.}\end{array}$ & $\begin{array}{l}1549 \\
1909 \\
2799 \\
3727\end{array}$ & $\begin{array}{l}3413 \pm 5 \\
4195 \pm 8 \\
6171 \pm 10 \\
8213 \pm 12\end{array}$ & $\begin{aligned} 84 & \pm 24 \\
78 & \pm 22 \\
150 & \pm 45 \\
40 & \pm 20\end{aligned}$ & $\begin{array}{l}1.7 \mathrm{e}-17 \\
4.3 \mathrm{e}-18 \\
9.5 \mathrm{e}-18 \\
7.9 \mathrm{e}-19\end{array}$ & $\begin{array}{r}21 \\
14 \\
16 \\
3.1\end{array}$ & 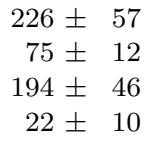 & abs on blue wing \\
\hline $\begin{array}{l}5 \mathrm{C} 7.118 \\
7 \mathrm{C} \mathrm{B} 081615.1+265130\end{array}$ & $\begin{array}{r}0.527 \\
\pm \\
.002\end{array}$ & $\begin{array}{l}\mathrm{Mg} \text { II } \\
{[\mathrm{Ne} \mathrm{V}]} \\
{[\mathrm{Ne} \mathrm{V}]} \\
\mathrm{H} \gamma \\
\mathrm{H} \beta \\
{[\mathrm{O}} \\
{[\mathrm{O}} \\
{[\mathrm{O}}\end{array}$ & $\begin{array}{l}2799 \\
3346 \\
3426 \\
4340 \\
4861 \\
4959 \\
5007\end{array}$ & $\begin{array}{l}4293 \pm 3 \\
5109 \pm 2 \\
5230 \pm 2 \\
6638 \pm 12 \\
7424 \pm 8 \\
7568 \pm 3 \\
7638 \pm 2\end{array}$ & $\begin{aligned} 59 & \pm 15 \\
19 & \pm 4 \\
15 & \pm 3 \\
115 & \pm 20 \\
120 & \pm 18 \\
27 & \pm 12 \\
17 & \pm 3\end{aligned}$ & $\begin{array}{l}7.5 \mathrm{e}-18 \\
8.8 \mathrm{e}-20 \\
9.0 \mathrm{e}-19 \\
1.2 \mathrm{e}-18 \\
3.4 \mathrm{e}-18 \\
2.1 \mathrm{e}-19 \\
6.4 \mathrm{e}-19\end{array}$ & $\begin{array}{r}31 \\
1.4 \\
14 \\
5 \\
9 \\
9.5 \\
8\end{array}$ & $\begin{array}{rr}65 \pm & 14 \\
2 \pm & 1 \\
14 \pm & 5 \\
29 \pm & \mathrm{f} 7 \\
89 \pm & 17 \\
5 \pm & 2 \\
17 \pm & 5\end{array}$ & cr in line \\
\hline $\begin{array}{l}5 \mathrm{C} 7.194 \\
7 \mathrm{C} \mathrm{B} 081914.4+254809\end{array}$ & $\begin{array}{r}1.738 \\
\pm \\
.005\end{array}$ & $\begin{array}{l}\text { Ly } \alpha / \text { NV } \\
\text { Si IV / O IV } \\
\text { C IV } \\
\text { He II } \\
\text { C III] } \\
\text { [Ne IV] } \\
\text { Mg II }\end{array}$ & $\begin{array}{l}1216 \\
1400 \\
1549 \\
1640 \\
1909 \\
2424 \\
2799\end{array}$ & $\begin{array}{l}3352 \pm 14 \\
3841 \pm 6 \\
4242 \pm 6 \\
4464 \pm 8 \\
5217 \pm 8 \\
6637 \pm 5 \\
7671 \pm 6\end{array}$ & $\begin{aligned} 92 & \pm 30 \\
97 & \pm 18 \\
85 & \pm 16 \\
99 & \pm 19 \\
100 & \pm 16 \\
35 & \pm 5 \\
52 & \pm 10\end{aligned}$ & $\begin{array}{l}8.9 \mathrm{e}-17 \\
1.0 \mathrm{e}-17 \\
1.5 \mathrm{e}-17 \\
3.3 \mathrm{e}-18 \\
1.0 \mathrm{e}-17 \\
5.9 \mathrm{e}-19 \\
4.0 \mathrm{e}-18\end{array}$ & $\begin{array}{r}32 \\
7 \\
19 \\
5 \\
16 \\
6 \\
6 \\
25\end{array}$ & 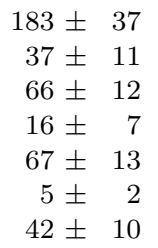 & $\begin{array}{l}\text { abs } \\
\text { abs }\end{array}$ \\
\hline
\end{tabular}

Table A1. (cont) 


\begin{tabular}{|c|c|c|c|c|c|c|c|c|c|}
\hline name & $z$ & line & $\begin{array}{c}\lambda_{\text {rest }} \\
(\AA)\end{array}$ & $\begin{array}{c}\lambda_{\text {obs }} \\
(\AA)\end{array}$ & $\begin{array}{c}\text { FWHM } \\
(\AA)\end{array}$ & $\begin{array}{c}\text { flux } \\
\left(\mathrm{W} \mathrm{m}^{-2}\right)\end{array}$ & $\begin{array}{c}\mathrm{snr} \\
\sigma\end{array}$ & $\begin{array}{l}\mathrm{W}_{\lambda} \\
(\AA)\end{array}$ & notes \\
\hline $\begin{array}{l}\text { 5C7.195 } \\
\text { 7C B081920.1+281538 }\end{array}$ & $\begin{array}{r}2.034 \\
\pm \\
.002\end{array}$ & $\begin{array}{l}\text { Ly } \alpha \\
\text { N V } \\
\text { Si IV / O IV } \\
\text { C IV } \\
\text { He II } \\
\text { C III] }\end{array}$ & $\begin{array}{l}1216 \\
1240 \\
1400 \\
1549 \\
1640 \\
1909\end{array}$ & $\begin{array}{l}3689 \pm 4 \\
3764 \pm 4 \\
4241 \pm 15 \\
4702 \pm 2 \\
4966 \pm 2 \\
5772 \pm 5\end{array}$ & $\begin{array}{l}34 \pm 4 \\
27 \pm 5 \\
40 \pm 20 \\
34 \pm 3 \\
21 \pm 4 \\
41 \pm 7\end{array}$ & $\begin{array}{l}3.9 \mathrm{e}-18 \\
1.4 \mathrm{e}-18 \\
3.3 \mathrm{e}-19 \\
8.5 \mathrm{e}-19 \\
3.0 \mathrm{e}-19 \\
9.2 \mathrm{e}-19\end{array}$ & $\begin{array}{r}23 \\
11 \\
5 \\
17 \\
10 \\
13\end{array}$ & 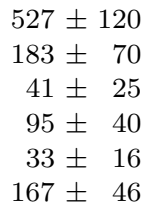 & $\begin{array}{l}\text { cr in line } \\
\text { abs } \\
\text { abs }\end{array}$ \\
\hline $\begin{array}{l}\text { 5C7.230 } \\
\text { 7C B082134.3+244829 }\end{array}$ & $\begin{array}{r}1.242 \\
\pm \\
.002\end{array}$ & $\begin{array}{l}\text { C IV } \\
\text { He II } \\
\text { C III] } \\
\text { C II] } \\
\text { Mg II }\end{array}$ & $\begin{array}{l}1549 \\
1640 \\
1909 \\
2326 \\
2799\end{array}$ & $\begin{array}{l}3468 \pm 6 \\
3674 \pm 5 \\
4274 \pm 8 \\
5222 \pm 2 \\
6276 \pm 4\end{array}$ & $\begin{array}{l}51 \pm 40 \\
52 \pm 40 \\
69 \pm 17 \\
25 \pm 5 \\
52 \pm 30\end{array}$ & $\begin{array}{l}1.2 \mathrm{e}-17 \\
1.7 \mathrm{e}-18 \\
2.0 \mathrm{e}-18 \\
3.1 \mathrm{e}-19 \\
1.5 \mathrm{e}-18\end{array}$ & $\begin{array}{r}15 \\
2.8 \\
7 \\
2.6 \\
7\end{array}$ & 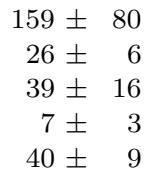 & $\begin{array}{l}\text { in noise } \\
\text { in noise } \\
\text { abs \& cr in line }\end{array}$ \\
\hline $\begin{array}{l}7 \mathrm{C} 0825+2930 \\
7 \mathrm{C} \mathrm{B} 082505.4+293018\end{array}$ & $\begin{array}{r}2.315 \\
\pm \\
.001\end{array}$ & $\begin{array}{l}\text { Ly } \alpha \\
\text { Si IV / O IV } \\
\text { C IV } \\
\text { C III] }\end{array}$ & $\begin{array}{l}1216 \\
1400 \\
1549 \\
1909\end{array}$ & $\begin{array}{l}4053 \pm 8 \\
4640 \pm 6 \\
5137 \pm 4 \\
6328 \pm 6\end{array}$ & $\begin{aligned} 127 & \pm 18 \\
80 & \pm 22 \\
144 & \pm 24 \\
186 & \pm 32\end{aligned}$ & $\begin{array}{l}9.0 \mathrm{e}-18 \\
9.1 \mathrm{e}-19 \\
5.7 \mathrm{e}-18 \\
2.9 \mathrm{e}-18\end{array}$ & $\begin{array}{r}30 \\
5 \\
43 \\
22\end{array}$ & $\begin{aligned} 654 & \pm 140 \\
67 & \pm 18 \\
440 & \pm 110 \\
287 & \pm 70\end{aligned}$ & $\begin{array}{l}\text { abs } \\
\text { abs }\end{array}$ \\
\hline
\end{tabular}

Table A1. (cont.) Table summarising the emission lines present in our optical spectra of the 7C quasars and BLRGs. Emission lines labelled 'abs' have absorption in them. Emission lines labelled 'atm abs' and 'cr in line' suffer from atmospheric absorption and cosmic ray hits, respectively. The line fluxes from the non-spectrophotometric observations in Table 2 have been scaled by up to 2.5 mag. to account for absorption by cloud. 


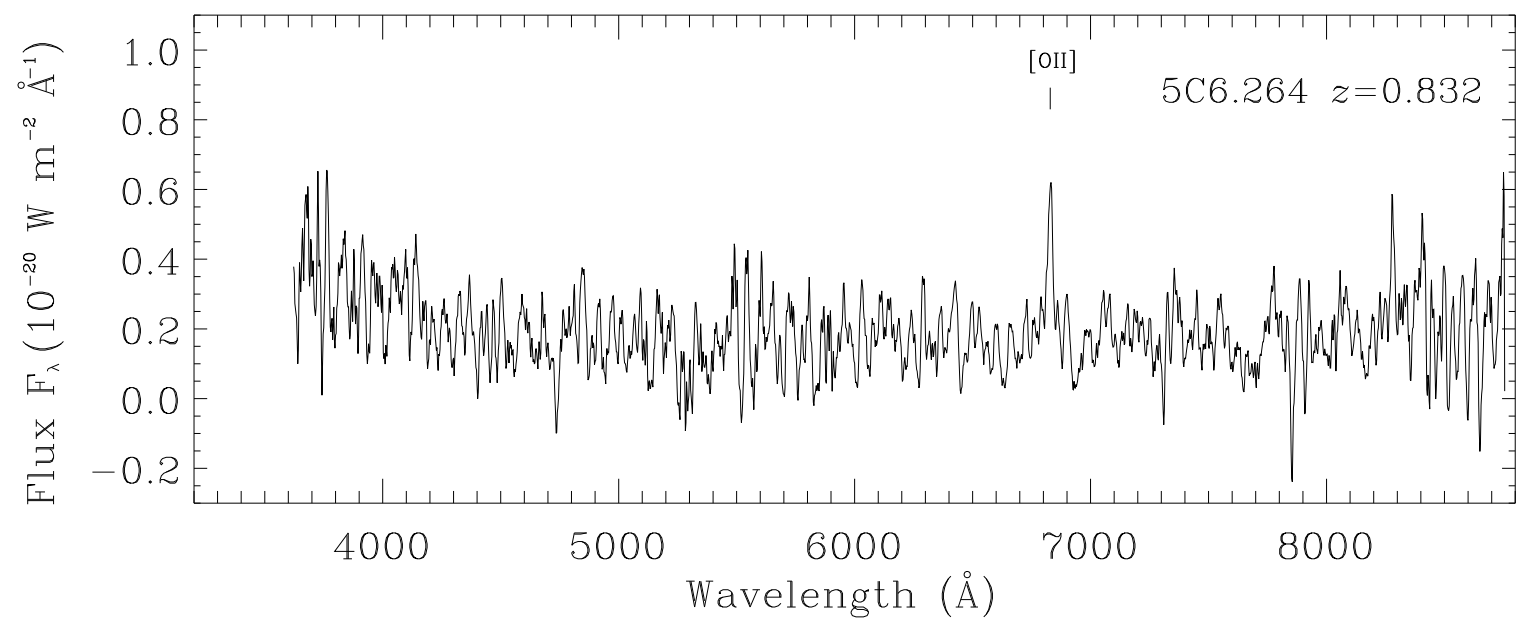

Figure A1. Optical spectrum of the quasar 5C6.264. This object was observed through cloud and the spectrum suffered several magnitudes of extinction. The spectrum has a blue continuum and a single narrow emission line which we identify as [OII] $\lambda 3727$ at a redshift of 0.832 . The $K$-band image of this object shows a very strong unresolved component and is $\sim 1$ mag brighter than the mean $K-z$ relation for radio galaxies (Eales et al. 1997). An infrared spectrum of 5C6.264 shows a broad H $\alpha$ line (Paper VIII). We attribute the lack of broad lines in the optical spectrum presented here (MgII $\lambda 2799$ expected at $5130 \AA)$ as solely due to its poor snr.

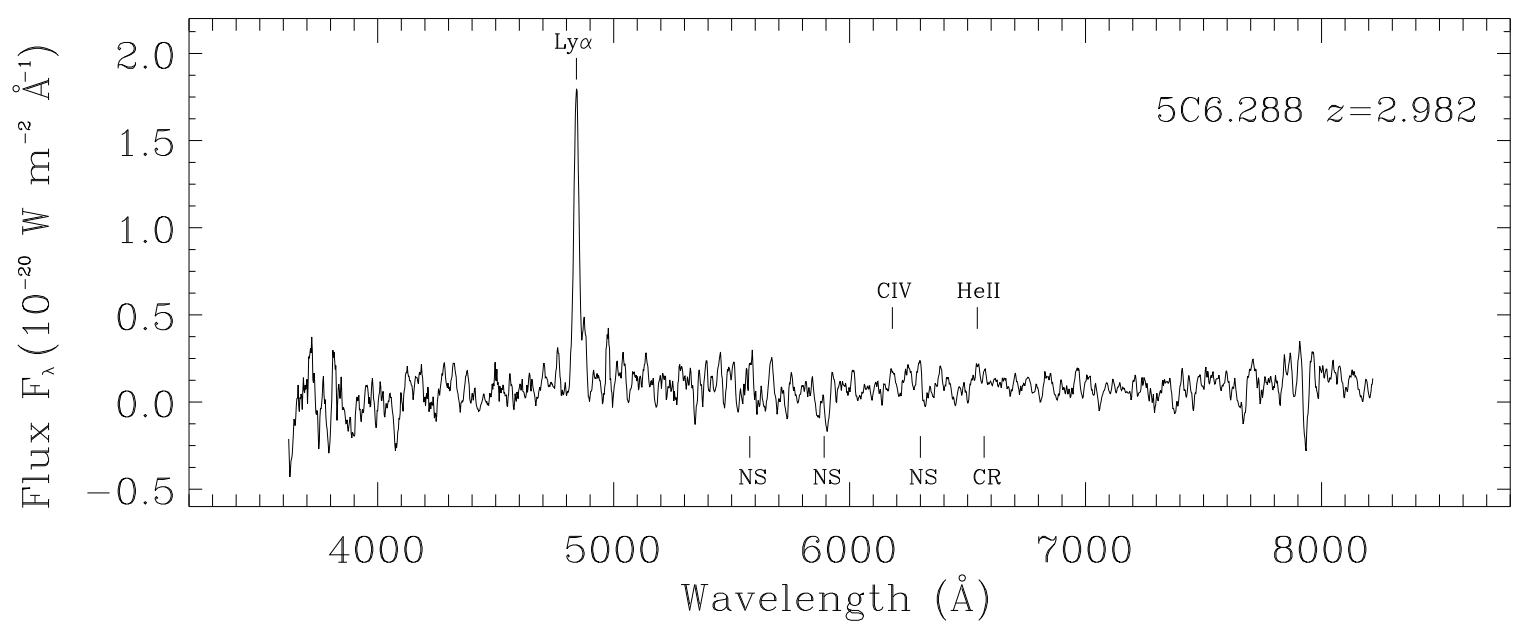

Figure A2. Optical spectrum of the probable quasar 5C6.288. This spectrum shows strong narrow Lyman- $\alpha$ emission and weak narrow CIV and HeII lines. The CIV and HeII lines are clearly visible on the 2-dimensional spectrum. The CIII] $\lambda 1909$ line is not observed as it falls in the atmospheric absorption band at $\sim 7600 \AA$. Regions of the spectrum affected by night sky lines and cosmic rays are marked NS and CR, respectively. The bright absolute magnitude $\left(M_{B}=-24.4\right)$ and compact nature at $K$-band are suggestive of a lightly reddened quasar $\left(A_{\mathrm{V}} \sim 1\right)$, which explains the lack of broad UV lines in the optical spectrum (see Paper VIII). 


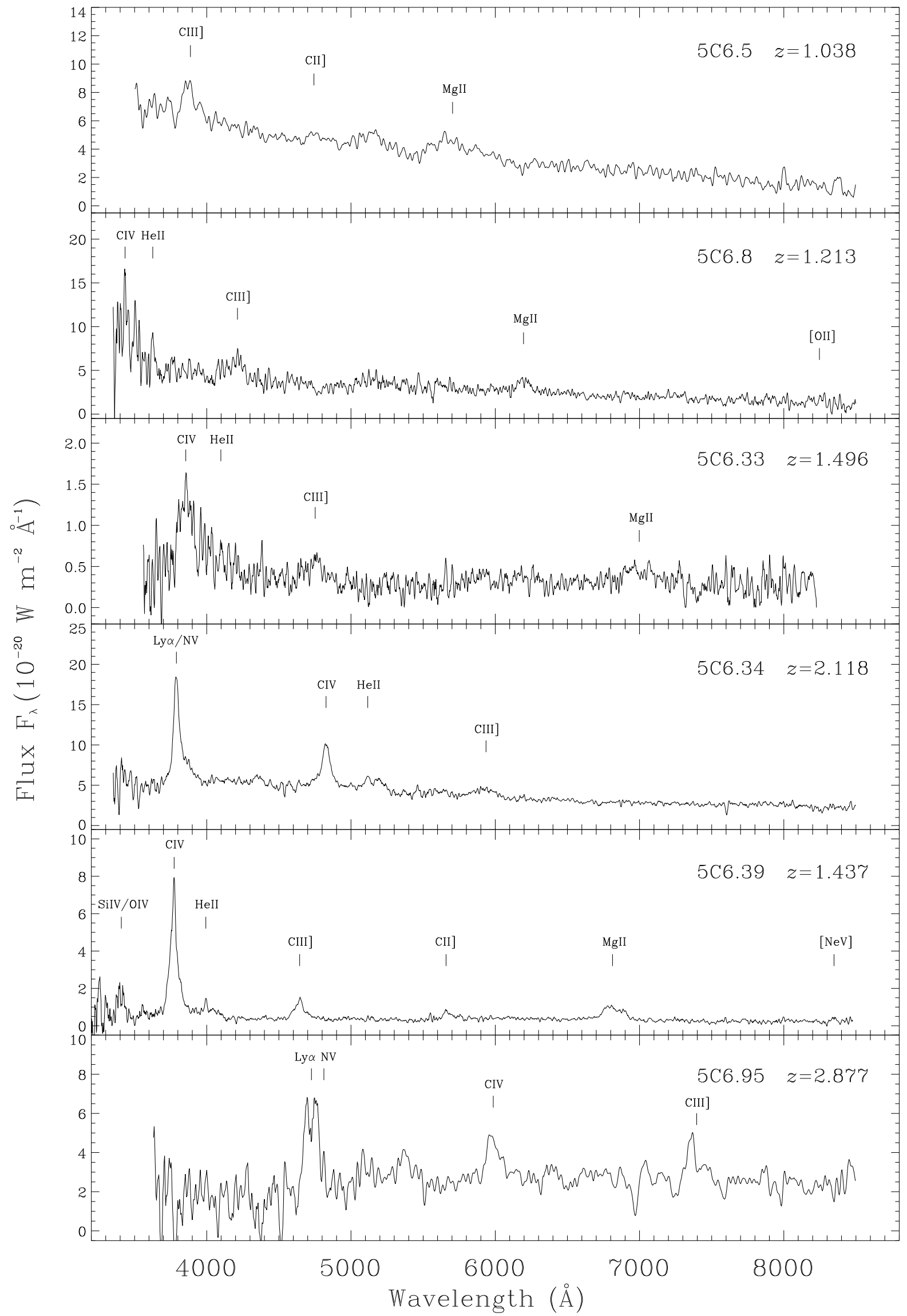

Figure A3. 


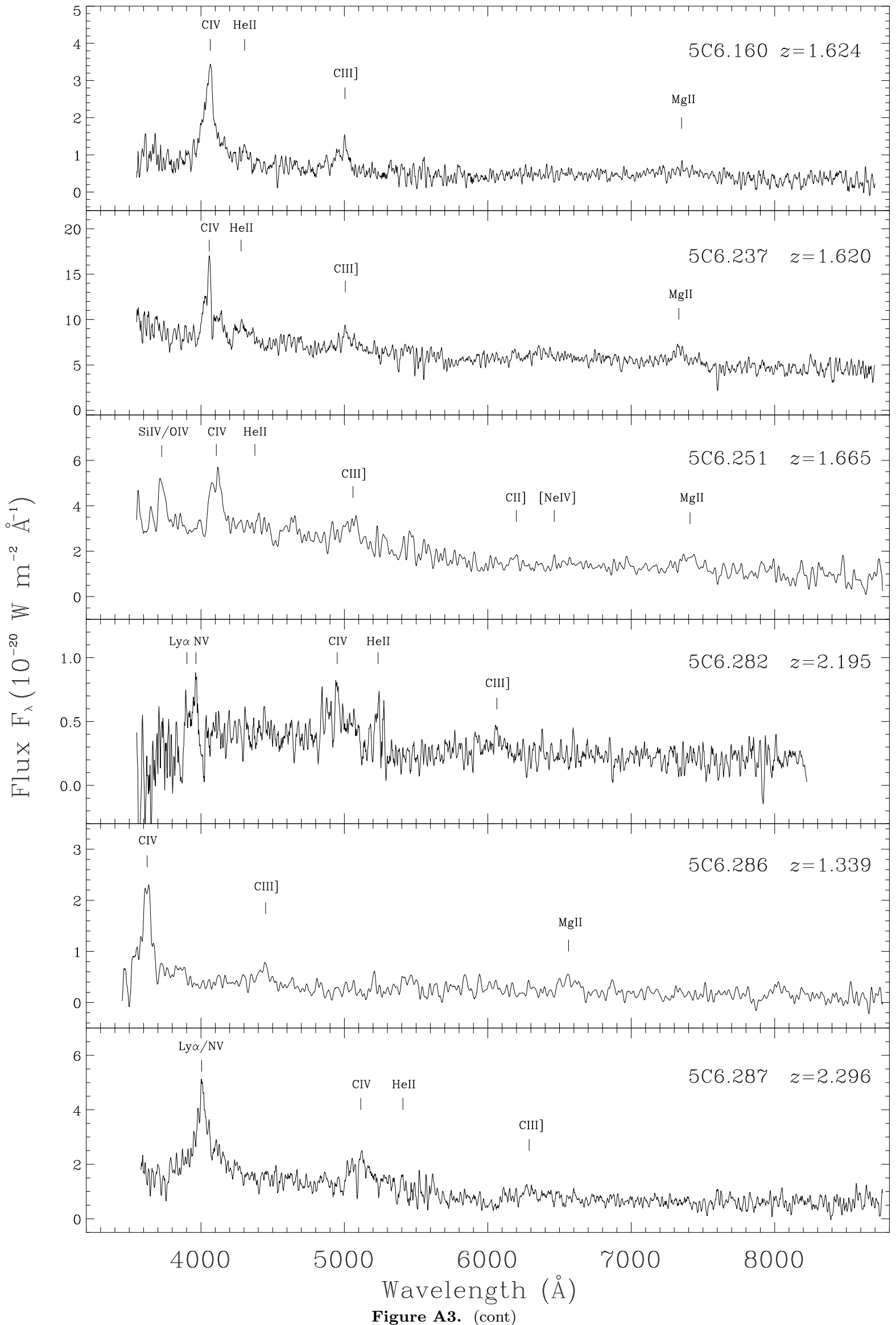

(c) 0000 RAS, MNRAS 000, 000-000 


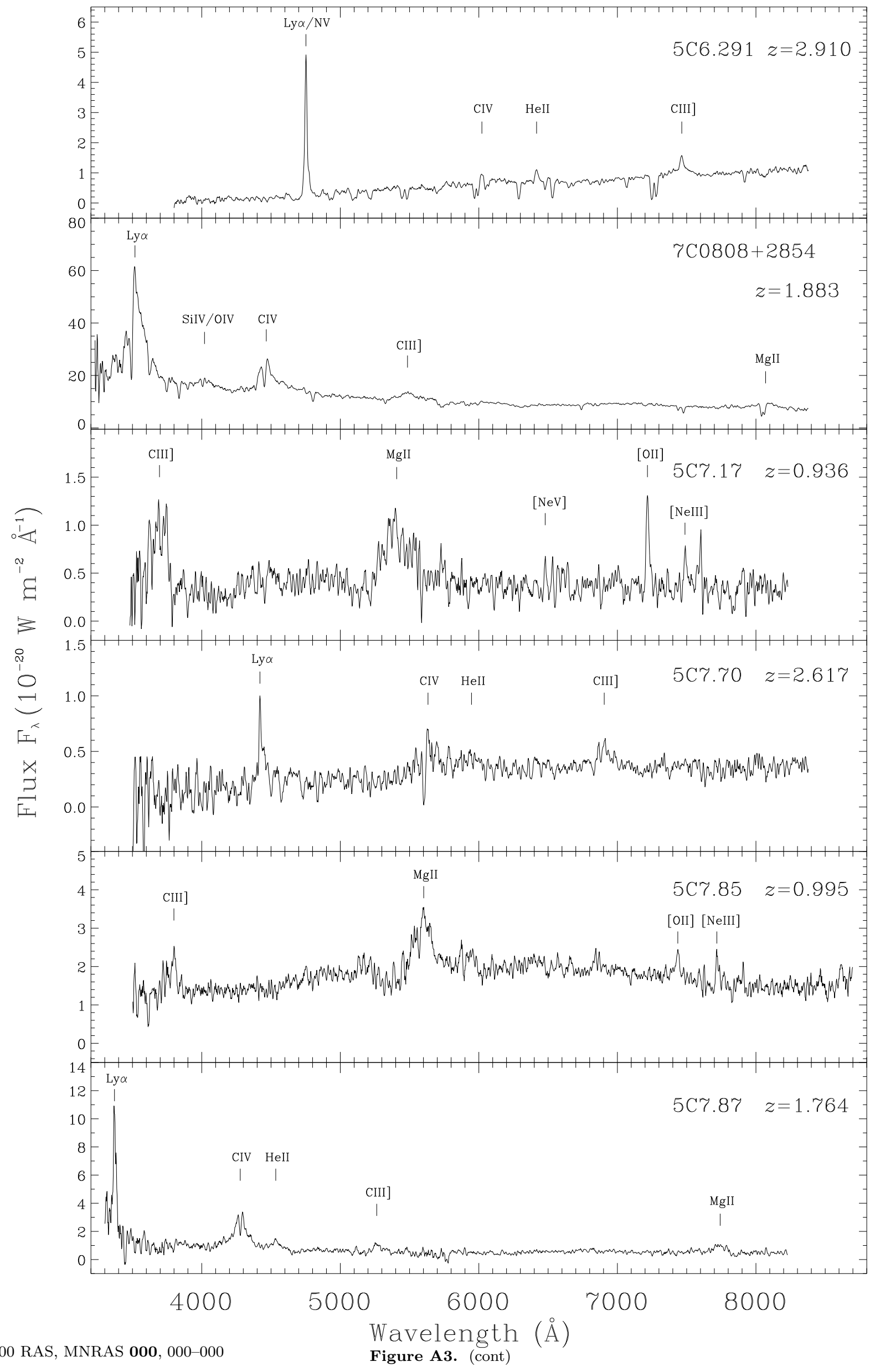




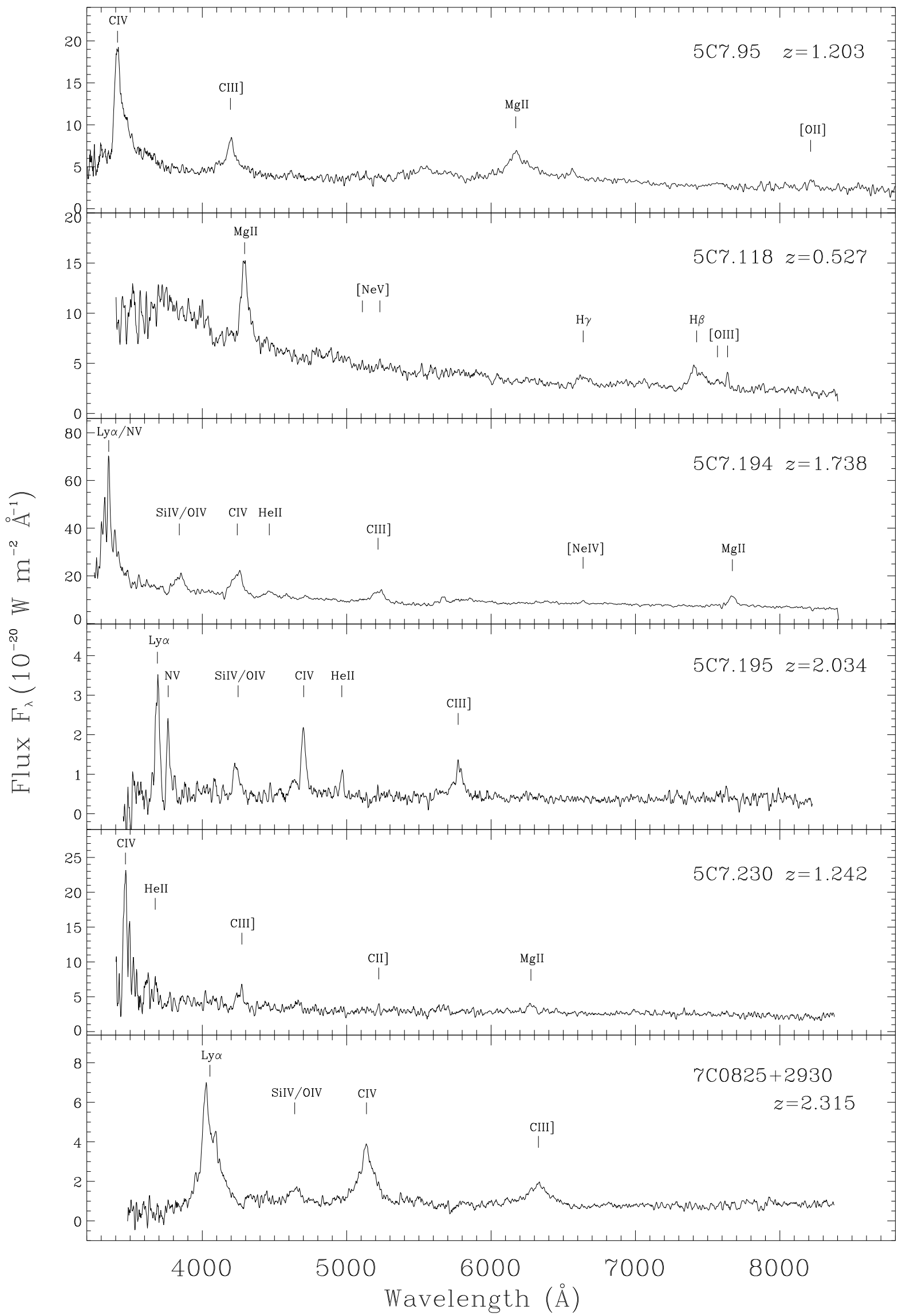

Figure A3. (cont) Optical spectra of quasars and broad-line radio galaxies in the $7 \mathrm{C}$ sample. 


\section{APPENDIX B: 3CRR QUASARS}

Table B1 lists the 3CRR quasar sample used in this paper which was combined with the $7 \mathrm{C}$ sample to give good coverage of the radio luminosity-redshift plane. Only two quasars (3C345 and 3C454.3) are excluded on the basis of having Doppler boosted cores/jets which raise their total lowfrequency flux above the sample limit. One quasar (3C286) has a rest-frame $1 \mathrm{GHz}$ spectral index $\alpha_{\text {rad }}<0.5$, but has enough extended flux to remain in the sample. Therefore it is called a core-jets quasar (CJS; see Section 2).

The full list of objects we classify as broad-line radio galaxies in the 3CRR sample is 3C22 (RLSE), 3C33.1 (LJWU), 3C41 (SRL), 3C61.1 (EH), 3C67 (LJWU), 3C219 (LJWU), 3C268.3 (LJWU), 3C303 (EH), 3C318 (SS), 3C381 (GO), 3C382 (EH), 3C390.3 (EH) \& 3C455 (SDMA). Key to broad-emission line references: EH - Eracleous \& Halpern (1994); GO - Grandi \& Osterbrock (1978); LJWU - Laing et al. (1994); RLSE - Rawlings et al. (1995); SDMA - Spinrad et al. (1985); SRL - Simpson, Rawlings \& Lacy (in prep.); SS - Spinrad \& Smith (1976) 


\begin{tabular}{|c|c|c|c|c|c|c|}
\hline Name & $\begin{array}{l}S_{178} \\
\text { (Jy) }\end{array}$ & $\alpha_{\mathrm{rad}}$ & $z$ & $\begin{array}{c}\log _{10}\left(L_{151}\right) \\
\left(\mathrm{W} \mathrm{Hz}^{-1} \mathrm{sr}^{-1}\right)\end{array}$ & $V$ & $M_{B}$ \\
\hline 3C9 & 19.402 & 1.08 & 2.012 & 28.68 & 18.21 & -26.60 \\
\hline 3C14 & 11.336 & 0.97 & 1.469 & 28.10 & 20.00 & -24.42 \\
\hline $3 \mathrm{C} 43$ & 12.644 & 0.76 & 1.457 & 28.09 & 20.00 & -23.70 \\
\hline $3 \mathrm{C} 47$ & 28.776 & 0.98 & 0.425 & 27.39 & 18.10 & -23.57 \\
\hline $3 \mathrm{C} 48$ & 59.950 & 0.71 & 0.367 & 27.46 & 16.20 & -25.27 \\
\hline 3С68.1 & 13.952 & 0.97 & 1.238 & 28.03 & 19.60 & -25.84 \\
\hline 3C109 & 23.544 & 0.79 & 0.305 & 26.96 & 17.88 & -23.14 \\
\hline $3 \mathrm{C} 138$ & 24.198 & 0.56 & 0.759 & 27.65 & 17.90 & -25.00 \\
\hline $3 \mathrm{C} 147$ & 65.945 & 0.60 & 0.545 & 27.80 & 16.90 & -25.43 \\
\hline $3 \mathrm{C} 175$ & 19.184 & 1.01 & 0.768 & 27.77 & 16.60 & -26.66 \\
\hline $3 \mathrm{C} 181$ & 15.805 & 0.93 & 1.382 & 28.16 & 18.92 & -25.75 \\
\hline $3 \mathrm{C} 186$ & 15.369 & 1.19 & 1.063 & 27.94 & 17.60 & -26.47 \\
\hline 3C190 & 16.350 & 0.88 & 1.197 & 28.06 & 20.00 & -23.98 \\
\hline 3C191 & 14.170 & 1.00 & 1.952 & 28.52 & 18.65 & -25.86 \\
\hline 3C196 & 74.338 & 0.88 & 0.871 & 28.38 & 17.60 & -26.24 \\
\hline $3 \mathrm{C} 204$ & 11.445 & 1.07 & 1.112 & 27.94 & 18.21 & -26.20 \\
\hline $3 \mathrm{C} 205$ & 13.734 & 1.00 & 1.534 & 28.23 & 17.62 & -27.46 \\
\hline $3 \mathrm{C} 207$ & 14.824 & 0.72 & 0.684 & 27.48 & 18.15 & -24.80 \\
\hline $3 \mathrm{C} 208$ & 18.312 & 1.07 & 1.109 & 28.06 & 17.42 & -26.49 \\
\hline $3 \mathrm{C} 212$ & 16.459 & 0.79 & 1.049 & 27.92 & 19.06 & -24.73 \\
\hline $3 \mathrm{C} 215$ & 12.426 & 1.02 & 0.411 & 26.98 & 18.27 & -23.35 \\
\hline $3 \mathrm{C} 216$ & 22.018 & 0.67 & 0.668 & 27.65 & 18.48 & -24.27 \\
\hline $3 \mathrm{C} 245$ & 15.696 & 0.73 & 1.029 & 27.88 & 17.25 & -26.73 \\
\hline $3 \mathrm{C} 249.1$ & 11.663 & 0.88 & 0.311 & 26.69 & 15.72 & -25.32 \\
\hline $3 \mathrm{C} 254$ & 21.691 & 1.05 & 0.734 & 27.74 & 17.98 & -24.75 \\
\hline $3 \mathrm{C} 263$ & 16.568 & 0.87 & 0.656 & 27.50 & 16.32 & -26.22 \\
\hline $3 \mathrm{C} 268.4$ & 11.227 & 0.90 & 1.400 & 28.01 & 18.42 & -26.72 \\
\hline $3 \mathrm{C} 270.1$ & 14.824 & 0.80 & 1.519 & 28.23 & 18.61 & -25.54 \\
\hline $3 \mathrm{C} 275.1$ & 19.947 & 0.82 & 0.557 & 27.52 & 19.00 & -23.26 \\
\hline $3 \mathrm{C} 286$ & 27.250 & 0.37 & 0.849 & 27.58 & 17.25 & -25.94 \\
\hline $3 \mathrm{C} 287$ & 17.767 & 0.52 & 1.055 & 27.79 & 17.67 & -26.78 \\
\hline 3C309.1 & 24.743 & 0.61 & 0.904 & 27.85 & 16.78 & -26.90 \\
\hline 3C334 & 11.881 & 0.90 & 0.555 & 27.25 & 16.41 & -25.78 \\
\hline $3 \mathrm{C} 336$ & 12.535 & 0.92 & 0.927 & 27.72 & 17.47 & -26.24 \\
\hline $3 \mathrm{C} 343$ & 13.516 & 0.68 & 0.988 & 27.60 & 20.61 & -23.27 \\
\hline 3C351 & 14.933 & 0.78 & 0.371 & 26.91 & 15.28 & -26.11 \\
\hline $4 \mathrm{C} 16.49$ & 11.445 & 1.00 & 1.296 & 28.05 & 18.50 & -25.62 \\
\hline 3C380 & 64.746 & 0.72 & 0.691 & 28.10 & 16.81 & -25.91 \\
\hline $3 \mathrm{C} 432$ & 11.990 & 1.07 & 1.805 & 28.36 & 17.96 & -26.61 \\
\hline $3 \mathrm{C} 454$ & 12.644 & 0.79 & 1.757 & 28.33 & 18.47 & -25.86 \\
\hline
\end{tabular}

Table B1. Data for the 3CRR quasar sample. The $178 \mathrm{MHz}$ fluxes are those listed in LRL multiplied by 1.09 , to correct for an error in the KPW flux scale at $178 \mathrm{MHz}$ (Roger et al. 1973). Spectral indices have been evaluated at $1 \mathrm{GHz}$ rest-frame. Rest-frame $151 \mathrm{MHz}$ luminosities are calculated from corrected $178 \mathrm{MHz}$ fluxes and spectral fitting as described in Blundell et al. (1998a). All $V$ magnitudes are taken from LRL and have not been corrected for galactic reddening. Absolute magnitudes were calculated as described in Section 5.1. $\mathrm{L}_{151}$ and $M_{B}$ assume $\Omega_{M}=1, \Omega_{\Lambda}=0$. 